\title{
Confirming the least massive members of the Pleiades star cluster
}

\author{
M. R. Zapatero Osorio, ${ }^{1 \star}$ V. J. S. Béjar, ${ }^{2,3 \star}$ N. Lodieu ${ }^{2,3 \star}$ and E. Manjavacas ${ }^{4}$ \\ ${ }^{1}$ Centro de Astrobiología (CSIC-INTA), Carretera Ajalvir km 4, E-28850 Torrejón de Ardoz, Madrid, Spain \\ ${ }^{2}$ Instituto de Astrofísica de Canarias, C/Vía Láctea s/n, E-38200 La Laguna, Tenerife, Spain \\ ${ }^{3}$ Universidad de La Laguna, E-38206 La Laguna, Tenerife, Spain \\ ${ }^{4}$ Department of Astronomy/Steward Observatory, The University of Arizona, 933 N. Cherry Avenue, Tucson, AZ 85721, USA
}

Accepted 2017 December 4. Received 2017 December 1; in original form 2017 October 31

\begin{abstract}
We present optical photometry ( $i$ and $Z$ band) and low-resolution spectroscopy (640-1015 nm) of very faint candidate members $(J=20.2-21.2 \mathrm{mag})$ of the Pleiades star cluster (120 Myr). The main goal is to address their cluster membership via photometric, astrometric, and spectroscopic studies, and to determine the properties of the least massive population of the cluster through the comparison of the data with younger and older spectral counterparts and state-of-the art model atmospheres. We confirm three bona fide Pleiades members that have extremely red optical and infrared colours, effective temperatures of $\approx 1150$ and $\approx 1350 \mathrm{~K}$, and masses in the interval 11-20 $M_{\text {Jup }}$, and one additional likely member that shares the same motion as the cluster but does not appear to be as red as the other members with similar brightness. This latter object requires further near-infrared spectroscopy to fully address its membership in the Pleiades. The optical spectra of two bona fide members were classified as L6-L7 and show features of $\mathrm{K}_{\mathrm{I}}$, a tentative detection of Cs I, hydrides, and water vapour with an intensity similar to high-gravity dwarfs of related classification despite their young age. The properties of the Pleiades L6-L7 members clearly indicate that very red colours of $\mathrm{L}$ dwarfs are not a direct evidence of ages younger than $\approx 100 \mathrm{Myr}$. We also report on the determination of the bolometric corrections for the coolest Pleiades members. These data can be used to interpret the observations of the atmospheres of exoplanets orbiting stars.
\end{abstract}

Key words: brown dwarfs - stars: late type - stars: low mass - open clusters and associations: individual: Pleiades.

\section{INTRODUCTION}

Understanding the evolution and physical properties of brown dwarfs and isolated planetary-mass objects is key for the interpretation of atmospheric observations of unseen planets orbiting stars. To build the picture of the substellar evolutionary sequence, various groups are carrying out deep photometric surveys on different nearby star-forming regions and young star clusters with ages ranging from $\approx 1$ though 800 Myr (e.g. Dawson, Scholz \& Ray 2011; Boudreault et al. 2012; Casewell et al. 2012, 2014; Lodieu, Deacon \& Hambly 2012; Peña Ramírez et al. 2012; Alves de Oliveira et al. 2013; Lodieu 2013; Downes et al. 2014; Luhman, Esplin \& Loutrel 2016; Esplin \& Luhman 2017; Luhman et al. 2017; Pérez-Garrido, Lodieu \& Rebolo 2017; Schneider et al. 2017). With an age of $120 \pm 10 \mathrm{Myr}$ (Basri, Marcy \& Graham 1996; Martín et al. 1998a; Stauffer, Schultz \& Kirkpatrick 1998), a distance of $133.5 \pm 1.2 \mathrm{pc}$ (Soderblom et al. 2005; Melis et al. 2014; Galli

^E-mail: mosorio@cab.inta-csic.es (MRZO); vbejar@iac.es (VJSB); nlodieu@iac.es (NL) et al. 2017), and more than 1000 known members (Sarro et al. 2014), the Pleiades star cluster offers a unique opportunity to scrutinize brown dwarfs and planetary-mass objects of known (intermediate) age and solar metallicity. Previous attempts to identify Pleiades substellar objects with $\mathrm{L}$ and T types were reported by Martín et al. (1998b), Bihain et al. (2006), Casewell et al. (2007, 2010), and Lodieu et al. (2012). Spectroscopic follow-up observations have confirmed L-type Pleiades brown dwarf members down to $\sim \mathrm{L} 4$ spectral type (Bihain et al. 2010), yet no T-type cluster object is unambiguously identified to date (see Casewell et al. 2011; Lucas et al. 2013), partly because these sources are intrinsically very dim and spectroscopic observations are challenging.

Our group discovered a population of 19 faint, red Pleiades candidates in an area of $0.8 \mathrm{deg}^{2}$ whose proper motions are compatible with the distinctive one of the cluster (Zapatero Osorio et al. 2014a). This survey covered about 3 per cent of the total area of the cluster and extended the known Pleiades photometric sequence by about 2 mag fainter than any previous photometric and astrometric exploration carried out on the cluster. If corroborated as Pleiades members, the faintest candidates may have temperatures below the onset of methane absorption and masses below the deuterium burning 
mass limit of $\approx 13 M_{\text {Jup }}$. In a follow-up paper, Zapatero Osorio et al. (2014b) reported on the near-infrared low-resolution spectroscopy of six candidates in the interval $J=17.5-20.3 \mathrm{mag}$, the photometric $Z$-band detection of Calar Pleiades $21(Z=22.91 \pm 0.15 \mathrm{mag})$, and an upper limit on the $Z$-band magnitude of Calar Pleiades 25 ( $Z \geq 23.35 \mathrm{mag}$ ). These observations confirmed the cool nature of all sources with near-infrared spectral types determined in the range L2-L7.

Here, we continue the follow-up optical observations of the six faintest Calar Pleiades ${ }^{1}$ candidates from Zapatero Osorio et al. (2014a): Calar 21-26. Their magnitudes are in the interval $J=20.2$ $21.2 \mathrm{mag}$, and their masses would range from 11 to $20 M_{\text {Jup }}$ if confirmed as cluster members. We report on new low-resolution spectroscopy at visible wavelengths for Calar 21 and 22, and the $Z$-band detections of Calar 22, 23, 24 (also detected in the $i$ band), 25 , and 26 . Using the new optical data of this paper and the nearinfrared spectra and near- and mid-infrared photometry published in Zapatero Osorio et al. (2014a,b), our objectives are twofold. We aim at robustly establishing their cluster membership via the confirmation of their proper motion measurements and their red spectrophotometric spectral energy distributions (SEDs) from optical to infrared wavelengths, and at discussing their photometric and spectroscopic properties in comparison with other young and old spectral counterparts. In Section 2, we present the new imaging and spectroscopic observations. The analysis of the cluster membership based on astrometric, photometric, and spectroscopic results is given in Section 3. The properties of the confirmed least luminous Pleiades members are discussed in Section 4, where we also consider the spectral fitting of the data using state-of-the art model atmospheres and the calculation of bolometric luminosities and masses. Section 5 presents the conclusions of this paper. In an appendix, we introduce new follow-up data of one high proper motion white dwarf discovered in Zapatero Osorio et al. (2014a).

\section{OBSERVATIONS}

All observations were acquired with the Optical System for Imaging and low-Intermediate-Resolution Integrated Spectroscopy (OSIRIS; Cepa et al. 2000), mounted on the Nasmyth focus of the 10.4-m Gran Telescopio de Canarias (GTC) on the Roque de los Muchachos Observatory. The detector of OSIRIS consists of two Marconi CCD42-82 (2048 $\times 4096$ pixels $)$ with a small gap between them and with a pixel pitch of $15 \mu \mathrm{m}$. We only used the second detector since it is less affected by vignetting. The standard photometric and spectroscopic observing modes use binned pixels with a size of 0'.25822 on the sky (Sahlmann et al. 2016). Table 1 provides the journal of the photometric and spectroscopic observations including the observing dates (Universal Time), exposure times, the airmass at the start of data acquisition, and the average seeing conditions. All nights were photometric; the typical seeing was of about 1 arcsec or better.

In addition to the Calar candidate members, we also obtained OSIRIS data of the high proper motion source NPM 03 (Zapatero Osorio et al. 2014a); the acronym 'NPM' stands for non-proper motion candidate member of the Pleiades. This object lies close to Calar 21 on the sky (both objects can be imaged simultaneously with OSIRIS) and has a proper motion twice as large as that of the cluster in the right ascension coordinate while its motion in the declination axis is similar to the Pleiades. We report on the observations of

${ }^{1}$ From now on, we will use the abridged name 'Calar'.
Table 1. Journal of photometric and spectroscopic observations with OSIRIS on the GTC. On-source exposure time is given as number of exposures multiplied by individual integration time.

\begin{tabular}{lccccc}
\hline Object & $\begin{array}{c}\text { Obs. date } \\
\text { (UT) }\end{array}$ & Fil./Grat. & $\begin{array}{c}\text { Exp. } \\
(\mathrm{s})\end{array}$ & Airmass & Seeing \\
\hline Calar 21 & 2014 Nov 24 & Sloan $z$ & $8 \times 90$ & 1.08 & $0^{\prime \prime} 9$ \\
Calar 21 & 2014 Nov 24 & R300R & $4 \times 2700$ & 1.04 & $0^{\prime \prime} 9$ \\
Calar 22 & 2014 Nov 25 & Sloan $z$ & $36 \times 30$ & 1.03 & $1^{\prime \prime} 0$ \\
Calar 22 & 2015 Dec 11 & R300R & $6 \times 2700$ & 1.01 & $1^{\prime \prime} 0$ \\
Calar 23 & 2015 Dec 12 & Sloan $z$ & $27 \times 60$ & 1.39 & $0^{\prime \prime} 9$ \\
Calar 24 & 2015 Dec 12 & Sloan $z$ & $27 \times 60$ & 1.25 & $0^{\prime \prime} 7$ \\
Calar 24 & 2015 Dec 12 & Sloan $i$ & $24 \times 90$ & 1.19 & $0^{\prime \prime} 9$ \\
Calar 25 & 2014 Nov 26 & Sloan $z$ & $36 \times 30$ & 1.00 & $1^{\prime \prime} 0$ \\
Calar 26 & 2015 Dec 12 & Sloan $z$ & $27 \times 60$ & 1.46 & $0^{\prime \prime} 7$ \\
NPM 03 & 2014 Nov 24 & Sloan $z$ & $8 \times 90$ & 1.08 & $0^{\prime \prime} 9$ \\
NPM 03 & 2016 Jan 11 & R300R & $3 \times 1800$ & 1.24 & $1^{\prime \prime} 2$ \\
\hline
\end{tabular}

NPM 03 in Tables 1 and 2 and briefly discuss this particular object in the Appendix.

\subsection{Photometry}

We collected optical images of Calar 21, 22, 23, 24, 25, and 26 using the Sloan $z$-band filter. Calar 24 was also observed in the Sloan $i$ band in order to have an additional optical colour that would help us understand whether it is an L- or a T-type source (Table 1). Total on-source exposure times ranged from 720 to $2160 \mathrm{~s}$ depending on the brightness of the target, observing filter, and seeing conditions. Individual images were acquired with short exposures (30-90 s) and with $\sim 10$ arcsec offsets, which allowed us to remove cosmic rays and the Earth's atmosphere contribution to the sky emission. Raw frames were bias subtracted and flat-field corrected. For debiasing, we used the overscan regions of the detector. For flat-fielding, we median combined all debiased images obtained during the night to produce a 'super-flat'. Individual frames were properly aligned and stacked to produce final deep $i$ - and $z$-band images of the science targets and standard sources.

All Calar objects were detected in the Sloan $z$-band filter with signal-to-noise $(\mathrm{S} / \mathrm{N})$ ratio of $\approx 7$ and higher at the peak of the point spread functions (PSFs). Calar 24 was detected with a weak $\mathrm{S} / \mathrm{N} \approx 4$ in the very deep $i$-band exposure. Calar 23, which was detected at the border of the $J$ and $H$ images in the proper motion study by Zapatero Osorio et al. (2014a), is resolved into an extense source in the OSIRIS data. Its full width at half-maximum (FWHM) is about 1 pixel larger than the average seeing of point-like sources. We thus discarded Calar 23 as a substellar candidate of the Pleiades cluster, and will not consider it further in this paper. All remaining Calar sources are unresolved in the $z$-band images and they remain as Pleiades candidate members.

Aperture and PSF photometry was obtained using the 'phot' package within the IRAF ${ }^{2}$ environment. We defined circular apertures of radii $\geq 4 \times$ FWHM in size for deriving instrumental magnitudes. Photometric calibration of the $z$-band instrumental magnitudes of our targets was performed using the Z-band data of bright sources in the OSIRIS field of view provided by the UKIRT Infrared Deep

\footnotetext{
2 IRAF is distributed by National Optical Astronomy Observatories, which is operated by the Association of Universities for Research in Astronomy, Inc., under contract with the National Science Foundation.
} 
Table 2. Photometry, proper motions, optical spectral types, and membership flag of Calar Pleiades candidates. The high proper motion source NPM03 is also listed. Calar 23 is not included because it is resolved using the OSIRIS data (see the text). The $J$ - and $K$-band magnitudes are taken from Zapatero Osorio et al. (2014a). $Z, J$, and $K$ are in the Vega system; $i$ magnitude is in the AB system.

\begin{tabular}{|c|c|c|c|c|c|c|c|c|}
\hline Object & $\begin{array}{c}J \\
(\mathrm{mag})\end{array}$ & $\begin{array}{c}K \\
(\mathrm{mag})\end{array}$ & $\begin{array}{c}Z \\
(\mathrm{mag})\end{array}$ & $\begin{array}{c}i \\
(\mathrm{mag})\end{array}$ & SpT & 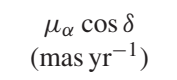 & $\begin{array}{c}\mu_{\delta} \\
\left(\operatorname{mas}_{\mathrm{yr}}^{-1}\right)\end{array}$ & Membership \\
\hline Calar 21 & $20.23 \pm 0.09$ & $17.73 \pm 0.03$ & $22.98 \pm 0.09$ & & L6-L7 & $+22.0 \pm 6.0$ & $-37.7 \pm 4.5$ & $\mathrm{Y}$ \\
\hline Calar 24 & $20.65 \pm 0.13$ & $18.83 \pm 0.33$ & $23.09 \pm 0.08$ & $25.80 \pm 0.19$ & & $+12.8 \pm 7.0$ & $-44.5 \pm 5.7$ & Y? \\
\hline Calar 25 & $20.83 \pm 0.15$ & $18.46 \pm 0.21$ & $23.79 \pm 0.10$ & & & $+23.4 \pm 8.2$ & $-47.9 \pm 6.8$ & $\mathrm{Y}$ \\
\hline Calar 26 & $21.15 \pm 0.14$ & $19.40 \pm 0.50$ & $23.71 \pm 0.10$ & & & $-0.2 \pm 10.0$ & $-25.3 \pm 15.0$ & $\mathrm{~N} ?$ \\
\hline
\end{tabular}

Sky Survey (UKIDSS; Lawrence et al. 2007), which fully overlaps with the Pleiades. The Sloan magnitudes were converted into the UKIDSS Z-band system using the colour terms appropriate for midto-late $\mathrm{L}$ types given by Hewett et al. (2006). The $i$-band data of Calar 24 was photometrically calibrated using OSIRIS observations of the field, young L5 dwarf 2MASS J03552337+1133437 (Reid et al. 2006), which was observed at similar air mass immediately after our target on the same night. We employed the Sloan magnitudes of the field L5 dwarf available from the Sloan Digital Sky Survey Data Release 11th and 12th (Alam et al. 2015). The final $i$ - (AB system) and Z-band (Vega system) magnitudes are listed in Table 2 .

The Z-band photometry of Calar 21 reported here agrees with the measurement published by Zapatero Osorio et al. (2014b) within less than $1 \sigma$ the quoted error bars. This hints to long time-scale photometric variability, if any, smaller than 0.15 mag in amplitude at these wavelengths. The actual $Z$ magnitude of Calar 25 (Table 2) also agrees with the upper limit reported in Zapatero Osorio et al. (2014b).

\subsection{Astrometry}

We used the OSIRIS images as the second epoch data to determine new proper motions for our targets following the methodology described in Zapatero Osorio et al. (2014a). This allowed us to confirm proper motions and identify sources with wrong astrometric measurements in the original paper. The time separation between the first epoch images ( $J$-band, pixel size of 0 '"3961, OMEGA' camera Zapatero Osorio et al. 2014a) and the OSIRIS data ranges from 16.07 to $17.12 \mathrm{yr}$. This is $1.8-1.9$ times longer than the time baseline of the first proper motion study, which would lead to more reliable astrometric determinations. In summary, the pixel coordinates of our targets and reference sources in the field were compared frame per frame and the coordinates transformations derived from the reference sources included third-order polynomials. Table 2 lists the proper motions of the Calar targets. Reported proper motion error bars correspond to the dispersion of the astrometric transformations, which included reference sources as bright and brighter than the targets. These errors are typically smaller by a factor of $\approx 1.3-2.0$ than those reported in table 1 by Zapatero Osorio et al. (2014a).

Calar 26 is the faintest source in the $J$ band and is located at $\sim 2$ arcsec south from another source, which may add 'noise' to its proper motion. To minimize this problem, we took advantage of the good seeing of the OSIRIS data (Table 1) and we derived the displacement of Calar 26 using both $J$ - and $H$-band images reported in Zapatero Osorio et al. (2014a) that were acquired 17.09 and $9.09 \mathrm{yr}$ before the OSIRIS image, respectively. The astrometric result given for Calar 26 in Table 2 corresponds to the averaged value of the two determinations. The two measurements coincide to better than $1 \sigma$ the quoted uncertainties.

\subsection{Optical spectroscopy}

We obtained the optical spectra of Calar 21 and 22, whose lowgravity nature was confirmed via near-infrared spectroscopy in Zapatero Osorio et al. (2014b), with two main objectives: the characterization of very red $\mathrm{L}$ dwarfs of well-known age and metallicity at various wavelengths, and the determination of bolometric luminosities, which are fundamental for deriving masses. Optical spectra were acquired using OSIRIS, the R300R grism, and the long 1arcsec slit. Together with the $2 \times$ binned pixels of the OSIRIS detector, this instrumental configuration delivers low-resolution spectra with a nominal dispersion of $7.8 \AA \mathrm{pix}^{-1}$ or a resolving power of $R \sim 270$ at $8500 \AA$, and wavelength coverage between 5000 and $12000 \AA$. The spectra were not acquired at parallactic angle since the targets and at least one bright reference star located at less than 1 arcmin distance were simultaneously aligned on the slit. This observational strategy allowed us to register and combine individual short exposures ( $2700 \mathrm{~s}$ each) taken in AB cycles (nodding positions were typically separated by $8-10$ arcsec for proper subtraction of the Earth's sky emission contribution). Observations were executed at or near culmination (see Table 1) and targets were acquired on the slit using the $z$-band filter (passband of 8 250-10000 ̊). Because our Calar candidates have very little flux at blue wavelengths $(\leq 6500 \AA)$, we do not expect significant light losses through the narrow slit due to Earth's relative atmospheric refraction. The nodding observations were repeated to yield on-source integrations of $3 \mathrm{~h}$ (Calar 21) and $4.5 \mathrm{~h}$ (Calar 22).

Raw images were reduced with standard procedures including bias subtraction and flat-fielding within IRAF. The registered frames were stacked together before optimally extracting the spectra of the targets. A full wavelength solution from calibration lamps taken during the observing nights was applied to the spectra. The error associated with the fifth-order Legendre polynomial fit to the wavelength calibration is typically 10 per cent of the nominal dispersion. We corrected the extracted spectra for instrumental response using data of the spectrophotometric standard white dwarf G191-B2B (Massey \& Gronwall 1990), which was observed on the same nights and with the same instrumental configuration as the targets. An order blocking filter blueward of $4500 \AA$ was used; however, there exists a second-order contribution redward of $9500 \AA$ (particularly important for blue objects), which was accounted for by observing the spectrophotometric standard star using the broad $z$-band filter and the same spectroscopic configuration as that of the science 


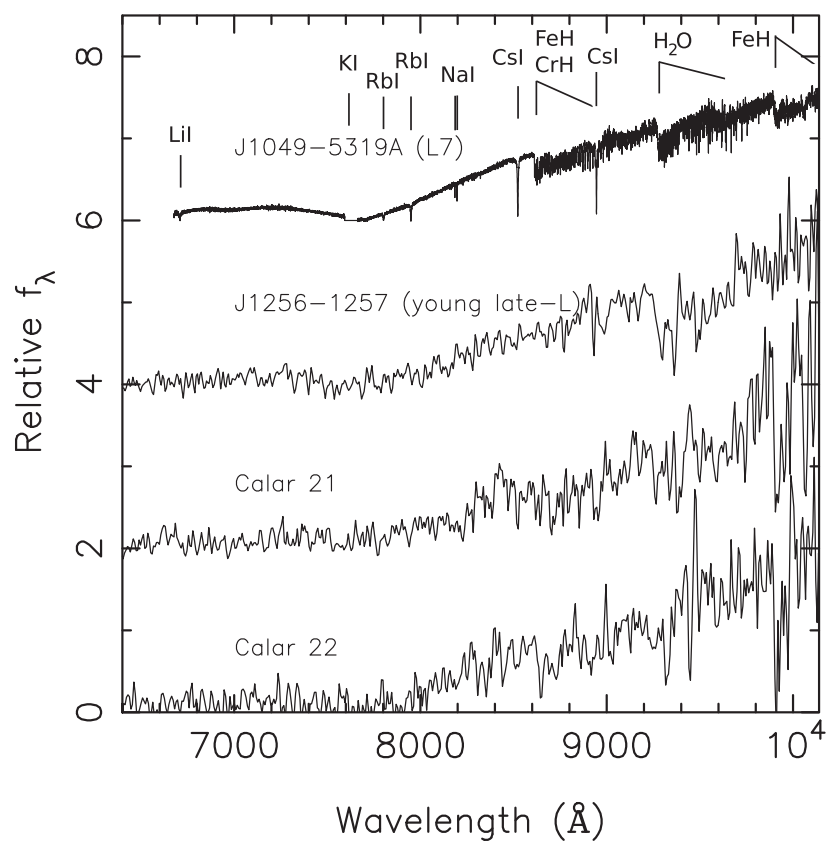

Figure 1. The OSIRIS optical spectra of Calar 21 and $22(R \approx 270)$ are shown together with the OSIRIS data of the young late-L dwarf $\mathrm{J} 1256-1257 \mathrm{~b}$ ( $R \approx 320$; Gauza et al. 2015). The top spectrum corresponds to the L8 high-gravity dwarf WISE J104915.57-531906.1A (Luhman 16A, Luhman 2013; Kniazev et al. 2013) obtained with X-SHOOTER $(R \approx 11000)$ and published in Lodieu et al. (2015). It is depicted to illustrate the location of the most relevant features at these wavelengths. Spectra are normalized to unity at $9050-9150 \AA$, and are shifted for clarity. Spectral features are identified after Kirkpatrick et al. (1999).

targets. To complete the data reduction, target spectra were divided by the standard star to remove the contribution of telluric absorption; the intrinsic features of the white dwarf were previously interpolated and its spectrum was normalized to the continuum before using it for division into the science data. The spectrophotometric standard star was observed a few hours before or after the targets; therefore, some telluric residuals may be present in the corrected spectra, particularly the strong $\mathrm{O}_{2}$ band at $7605 \AA$.

The resulting reduced OSIRIS spectra of Pleiades candidates Calar 21 and 22 are depicted in Fig. 1. The panel displays the wavelength range 6400-10150 $\AA$ because at shorter and longer wavelengths, the data have very poor $\mathrm{S} / \mathrm{N}$, and the instrumental response correction and wavelength calibration are not reliable at values beyond $\approx 1 \mu \mathrm{m}$. Together with the science targets, we also show the spectrum of the young, dusty L7 dwarf VHS J125601.92-125723.9b (Gauza et al. 2015; Stone et al. 2016), which was acquired with the same instrumentation and has comparable spectral resolution $(R \approx 320)$ as our data (spectrum published in Gauza et al. 2015). The spectra of the Calar sources and VHS J125601.92-125723.9b (J1256-1257b) are alike with peaks of cross-correlation functions of 0.96 (Calar 21 and J1256-1257b) and 0.92 (Calar 22 and J1256-1257b). The object J1256-1257b is believed to be 150-300 Myr old. The high-resolution spectrum $(R$ $\approx 11000$ ) of the field late-L dwarf WISE J104915.57-531906.1A (known as Luhman 16A, L8 according to Luhman 2013 and Kniazev et al. 2013; L7.5 according to Faherty et al. 2014; and L6-L7.5 according to Lodieu et al. 2015) obtained with the XSHOOTER instrument (see Lodieu et al. 2015) is also depicted in Fig. 1 for illustrating the location of the most relevant atomic and molecular features at red-optical wavelengths. This field ob-

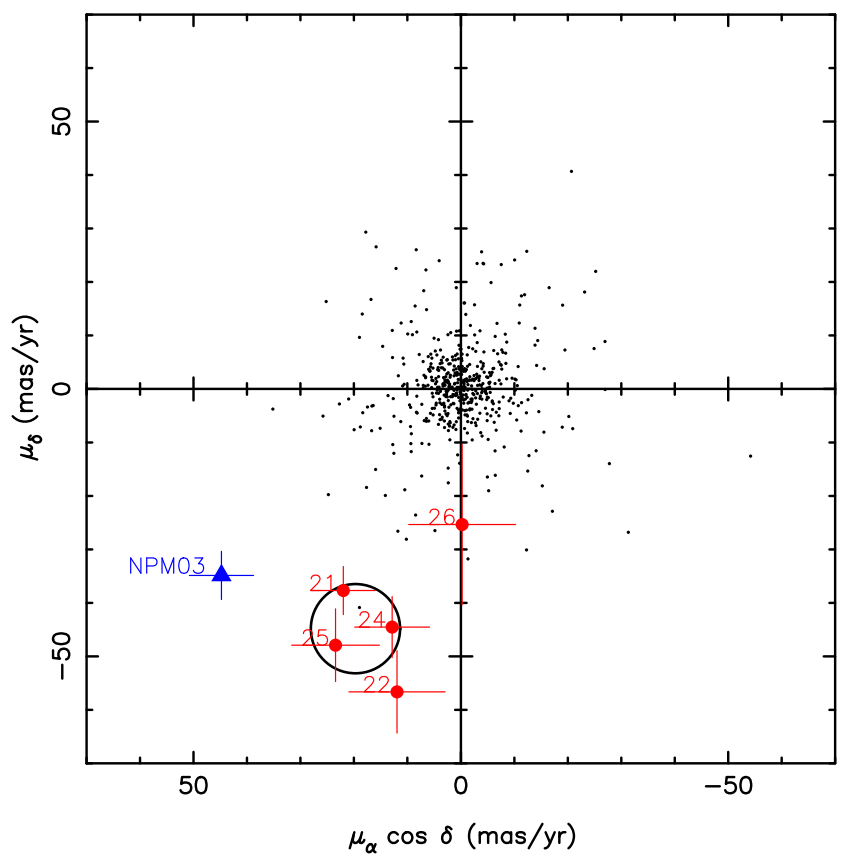

Figure 2. Proper motion diagram of the Pleiades candidates Calar 21, 22, 24,25 , and 26 (red dots). The mean motion of the stellar cluster lies at the centre $\left[(19.71,-44.82)\right.$ mas $^{-1}{ }^{-1}$; Loktin \& Beshenov 2003] of the black circle of radius of 8 mas $^{-1}$. The size of the circle corresponds to $1 \sigma$ astrometric error bar of sources with the same magnitudes as our targets. The tiny black dots around $(0,0)$ stand for the sample of objects contained within the field of view of the OSIRIS/GTC images and in common with the first epoch data. Our targets are labelled in red with their Calar identification number. The high proper motion source NPM 03 (see the Appendix) is plotted as a blue triangle.

ject likely has an age of $\approx 1$ Gyr (Faherty et al. 2014; Lodieu et al. 2015).

\section{CLUSTER MEMBERSHIP}

We revisited Calar 21, 22, 24, 25, and 26 membership in the Pleiades cluster using the OSIRIS photometry, astrometry, and spectroscopy. The last column of Table 2 summarizes our conclusion on cluster membership for each candidate after this paper.

\subsection{Proper motions}

Fig. 2 shows the proper motion diagram resulting from the astrometric study presented here. The location of Pleiades members is given by the black circle, which is centred at the mean proper motion of the cluster (Loktin \& Beshenov 2003) and has a radius equal to the average $1 \sigma$ uncertainty of our study. As seen from Fig. 2, Calar 21, 22, 24, and 25 lie close to the Pleiades motion, all except Calar 26. The new proper motion of Calar 26, although noisy, differs by more than $1 \sigma$ the quoted uncertainties with respect to the astrometry of Zapatero Osorio et al. (2014a); however, both measurements are compatible at the $2.5 \sigma$ level. Therefore, we consider that the membership of this object in the cluster has low probability. It is possible that the proper motion of Calar 26 is affected by the proximity of another source unrelated to the cluster. We confirm the astrometry of the remaining Calar objects: it coincides within $1 \sigma$ with the proper motions listed in Zapatero Osorio et al. (2014a). Calar 21, 22, 24, and 25 are proper motion candidate members of the Pleiades. 


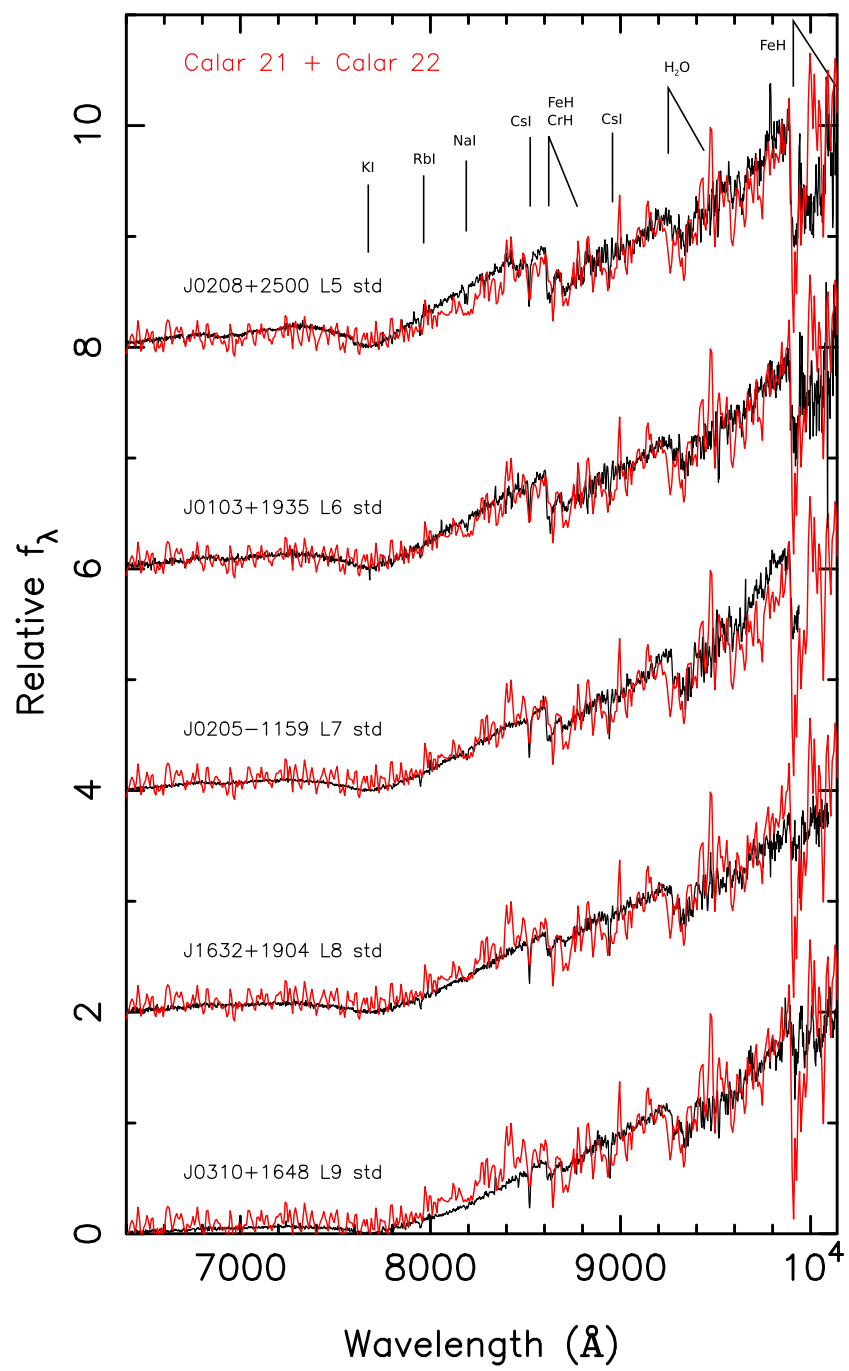

Figure 3. The combined OSIRIS spectrum of Calar 21 and 22 (red) is compared to high-gravity L5-L9 primary spectral standards (black) for classification. All spectra are normalized to unity at 9050-9150 A. Data are shifted by a constant in the vertical axis for clarity. From top to bottom, dwarf standards are the following: 2MASS J02085499+2500488 (L5), 2MASS J01033203+1935361 (L6), DENIS-P J0205.4-1159 (L7), 2MASS J16322911+1904407 (L8), and 2MASS J03105986+1648155 (L9) from the catalogues of Delfosse et al. (1997) and Kirkpatrick et al. $(1999,2000)$. The most relevant spectral features are indicated following Kirkpatrick et al. (1999).

\subsection{Spectral types}

We determined the spectral type of Calar 21 and 22 at optical wavelengths by direct comparison with data from the literature. Both spectral standard dwarfs defined by Kirkpatrick et al. (1999, 2000) and 'dusty' dwarfs were employed for this task. To increase the S/N of the observed data, we combined the OSIRIS spectra of Calar 21 and 22 into one single spectrum. This is justified by the similarity of Calar 21 and 22 properties from visible to infrared wavelengths (see Fig. 1, Table 2, and colour-magnitude diagrams discussed in next sections). The merged spectrum is shown against spectral standards ranging from L5 to L9 in Fig. 3. The optical spectra of the primary standard L dwarfs for spectral classification shown here were taken from Delfosse et al. (1997) and Kirkpatrick et al. (1999, 2000).

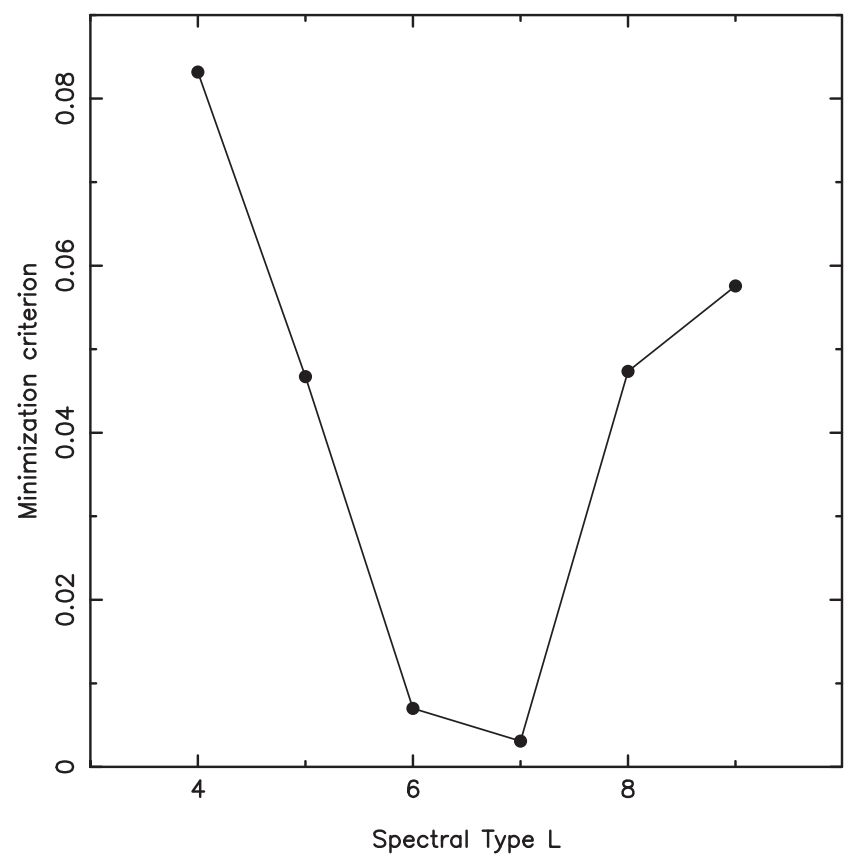

Figure 4. Figure of merit employed for the spectral classification of Calar 21 and 22. The minimum is located between L6 and L7.

These spectra ${ }^{3}$ were collected with the Low-Resolution Imaging Spectrograph (LRIS; Oke et al. 1995) at the $10 \mathrm{~m} \mathrm{W.} \mathrm{M.} \mathrm{Keck} \mathrm{Ob-}$ servatory on Mauna Kea, Hawaii, and have a resolution of $9 \AA$ over the wavelength range 6300-10 $100 \AA$. This is a factor of $\approx 3$ higher spectral resolution than our data; yet, it is acceptable for graphical purposes (Fig. 3).

We searched for the spectral primary standard that best resembles the Calar 21+22 combined spectrum at 'all' optical wavelengths. The following figure of merit (or minimization function) was defined:

merit $=\frac{1}{n} \sum_{i=1}^{n}\left|\left(f_{\mathrm{obs}}-f_{\mathrm{std}}\right)_{\lambda_{i}}\right|$,

where $f_{\text {obs }}$ and $f_{\text {std }}$ correspond to the fluxes of the OSIRIS/GTC and LRIS/Keck spectra at wavelengths $\lambda_{i}$, and $n$ stands for the total number of wavelengths explored in the interval 6400-9880 $\AA$ at the matching wavelengths of our data. The spectra of the standard dwarfs were resampled to the resolving power of the OSIRIS data. The resulting figure of merit is illustrated in Fig. 4. The minimum value of the figure corresponds to the best spectral resemblance. At optical wavelengths, Calar 21 and 22 are classified as L6-L7 dwarfs. Note that the absolute minimum is located at L7 in Fig. 4. However, the visual inspection of Fig. 3 indicates that the L6 typing cannot be easily discarded. Other minimization functions, like the goodnes-of-fit statistic function described in Cushing et al. (2008), show a broader minimum suggesting a spectral type in the interval L5-T0 (we used L and T standards and assigned equal weight to all wavelengths).

This optical classification is consistent with the near-infrared spectral typing of Zapatero Osorio et al. (2014b): L7 \pm 1 (Calar 21) and L/T (Calar 22), which was assigned based on the comparison of the infrared $H$ - and $K$-band data to spectra of young, dusty $\mathrm{L}$ dwarfs. However, the optical types appear to be slightly earlier

\footnotetext{
${ }^{3}$ http://www.stsci.edu/ inr/ultracool.html
} 
than the near-infrared ones; this signature is found among young ultracool dwarfs and has been mentioned by, e.g. Allers \& Liu (2013), Zapatero Osorio, Béjar \& Peña Ramírez (2017), and Lodieu et al. (2017). Particularly, Allers \& Liu (2013) discussed that their classification of young L dwarfs of the field and star moving groups shows an offset of $\approx 1$ subtype between infrared and optical spectral types. This phenomenon might be related to the low-gravity atmospheres of our targets and the impact of dust absorption at short wavelengths. Calar 21 and 22 have optical and near-infrared spectral types expected for Pleiades members with magnitudes around $K=18 \mathrm{mag}$; they follow the spectral type-magnitude relationships of Dupuy \& Liu (2012), Faherty et al. (2016), and Liu, Dupuy \& Allers (2016). In addition, Zapatero Osorio et al. (2014b) discussed the low-gravity features observed in the near-infrared spectra of Calar 21 and 22, and concluded that these two objects are spectroscopically confirmed members of the cluster.

\subsection{Colour-magnitude diagrams}

We used the $i$ - and Z-band photometry to assess the cluster membership of the Calar sources. In Fig. 5, we show the $K$ versus $Z-K$ (top panel) and $J$ versus $Z-J$ (bottom panel) colour-magnitude diagrams including previously known, confirmed Pleiades members with spectral types mid-M through mid-L, and our candidates. All magnitudes are given in the same photometric system allowing a direct comparison. Calar 21, 22, and 25 nicely extrapolates the cluster sequence towards fainter $J$ - and $K$-band magnitudes and redder $Z-K$ colours. As illustrated in the bottom panel, the $Z-J$ colours of the Calar candidates do not appear redder than those of brighter cluster members. Yet, Calar 25 is the reddest object in our sample; it has $Z-J=3.19 \pm 0.20$ and $Z-K=5.33 \pm 0.23$ mag. These indices are comparable to other very red, dusty late-L dwarfs like J1256-1257b, which is also plotted in Fig. 5 (J1256-1257b was taken to the distance of the Pleiades by using the new trigonometric parallax determination of $15.8_{-0.8}^{+1.0} \mathrm{pc}$ determined by Zapatero Osorio et al., in preparation).

Calar 24, although dimmer in $K$ band than Calar 25, has bluer $Z-K$ and $J-K$ colours and deviates by about 1 and $0.5 \mathrm{mag}$, respectively, from the increasingly red sequence of the cluster (see Figs 5 and 8). Whether this is evidence of the photometric nonmembership of Calar 24 in the Pleiades remains to be proved. On the one hand, Calar 26, whose proper motion indicates it is not a likely cluster member (Section 3.1), has $Z-K$ and $J-K$ colours similar to Calar 24. This may suggest that Calar 24 is a contaminant in the proper motion survey. On the other hand, in a recent work by Vos, Allers \& Biller (2017), it is discussed that the viewing angle influences the spectral and photometric appearance of ultracool, dusty dwarfs: objects viewed equator-on appear redder than objects viewed at lower inclinations. This would imply a large dispersion in the colours and magnitudes of coeval, equal mass objects. In addition, the turn-over sequence that is expected at optical and nearinfrared colour-magnitude diagrams and is caused by the onset of methane absorption and/or dust settlement below the photosphere in ultracool atmospheres, should occur at magnitudes near the position of Calar 25 in the colour-magnitude diagrams. Calar 24 occupies a position in Figs 5 and 8 well matched by the expected location of the 'methane sequence' in the Pleiades. Furthermore, Calar 24 shows $i-J$ and $i-Z$ colours similar to SDSS J085834.42+325627.6, which is a T0-T1 dwarf that displays redder than normal colours for its given spectral type (Faherty et al. 2009; Gagné et al. 2014). However, the kinematic study of Gagné et al. (2014) indicates that this $\mathrm{T}$ dwarf does not belong to any well-defined young star mov-
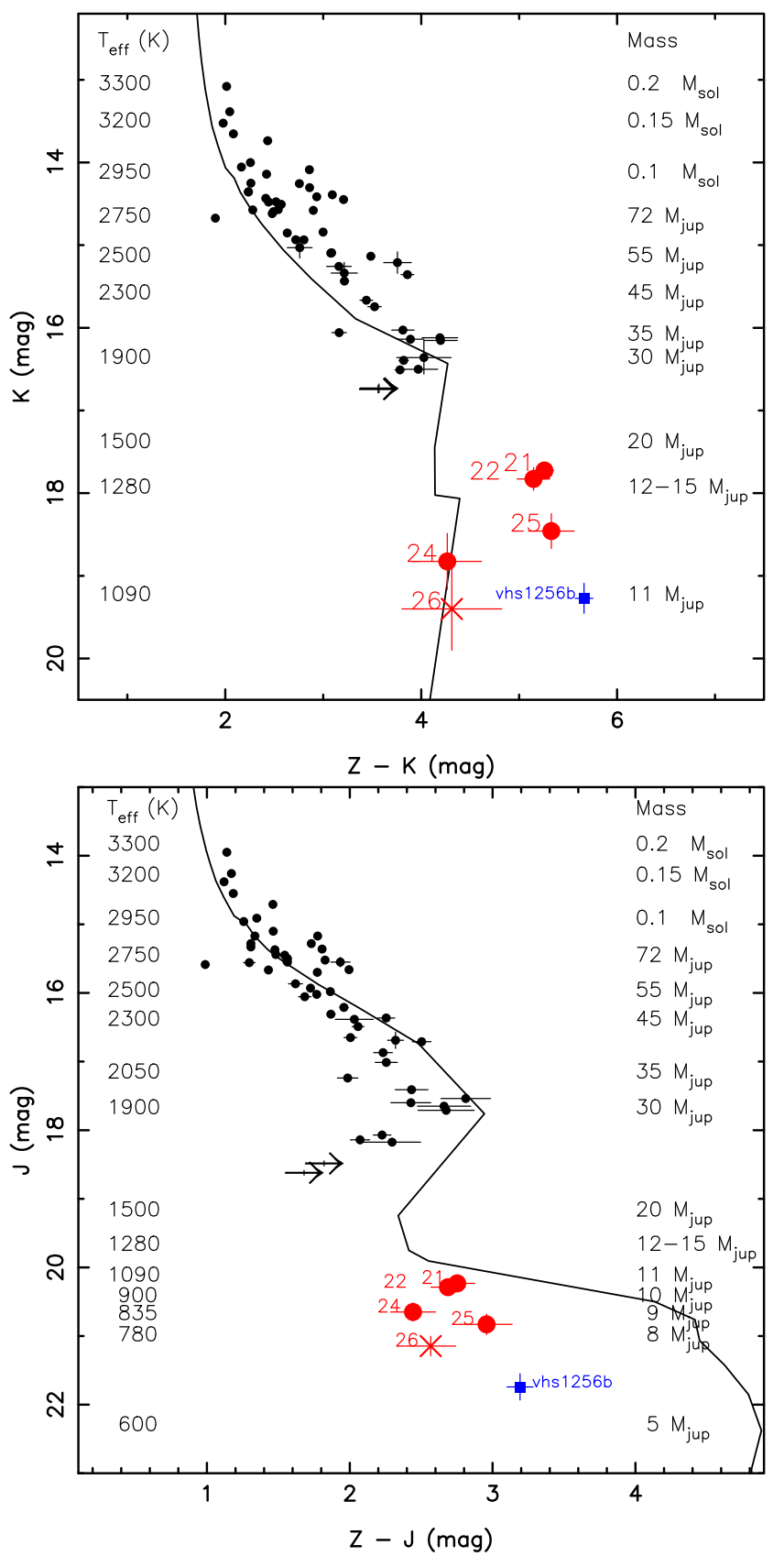

Figure 5. Colour-magnitude diagrams of Pleiades Calar candidates (red dots) using observed MKO photometry. The red asterisk corresponds to the likely non-member of the cluster. The Calar sources are labelled in red with their identification number. Previously known cluster members (Bihain et al. 2010; Zapatero Osorio et al. 2014a) are depicted with black dots; the arrows indicate Z-band upper limit magnitudes. The BT-Settl 120-Myr, solar metallicity isochrone (Allard 2014; Baraffe et al. 2015) is plotted as a solid line. Predicted masses and effective temperatures are labelled on the righthand and left-hand sides of the diagrams. The companion J1256-1257b, which has a likely age in the interval 150-300 Myr (Gauza et al. 2015), is shown in blue colour; it was taken to the distance of the Pleiades cluster.

ing group (TW Hydrae, $\beta$ Pictoris, Tucana-Horologium, Carina, Columba, Argus, and AB Doradus) and has a low probability of being a member of the young field. In colour-colour diagrams, like the one illustrated in Fig. 6, Calar 24 does not stand out as a $\mathrm{T}$ dwarf; early-Ts and Ls have some overlapping properties in various colours. At present, and with current astrometric and 


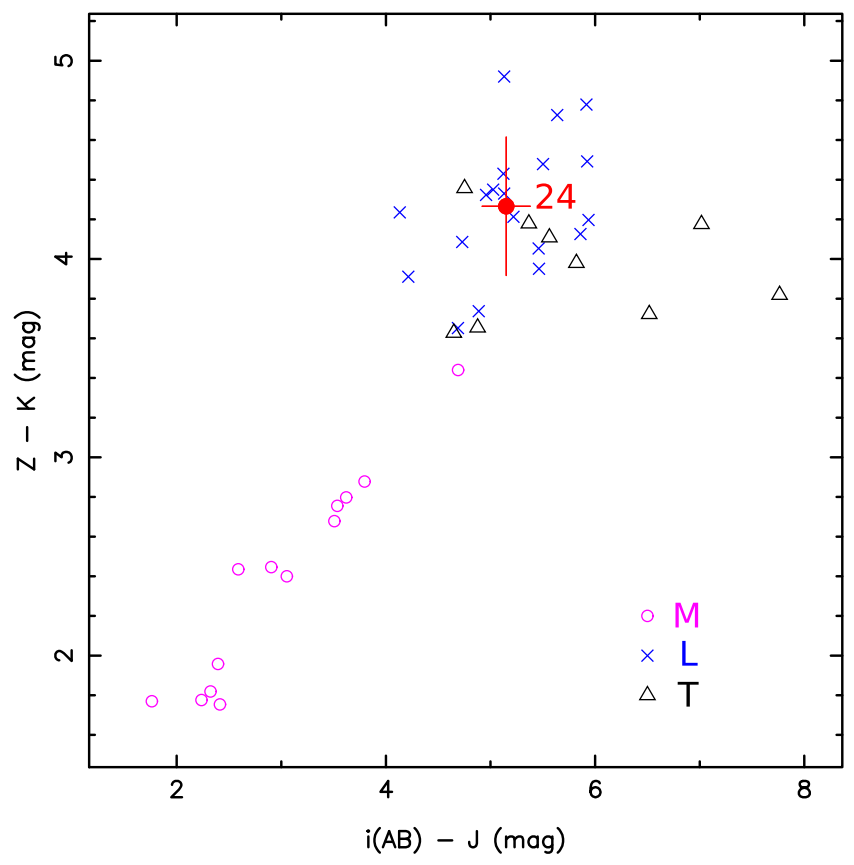

Figure 6. Colour-colour diagram to illustrate the $(i-J, Z-K)$ indices of Calar 24 (red dot) with respect to field M (open circles), L (crosses), and T (open triangles) dwarfs listed in Hewett et al. (2006). L and early-T sources show significant overlap, thus impeding the unambiguous spectrophotometric classification of Calar 24 with current photometric data.

photometric data, Calar 24 cannot be rejected as a Pleiades candidate member, but it cannot be confirmed either. The cluster membership flag of Calar 24 in the last column of Table 2 includes a '?' to remind that this object's membership status is still open. Spectroscopic observations at near-infrared wavelengths are required to explore the presence of methane absorption and other features typical of cool, low-gravity atmospheres that would unambiguously confirm/reject Calar 24 as a Pleiades member and would contribute to characterize the 'methane sequence' of the cluster.

\section{DISCUSSION}

\subsection{Spectral properties}

At the spectral resolution and quality of our data, the following spectroscopic features are seen in the optical spectrum of Calar $21+22$ (Fig. 3): the broad line of the resonance doublet of neutral potassium, K I, centred at 7665 and $7699 \AA$, molecular absorptions due to metallic hydrides $\mathrm{FeH}(\approx 8692,9896 \AA)$ and $\mathrm{CrH}(\approx 8611 \AA)$, and absorption due to water vapour $(\approx 9300 \AA)$. All of them show intensities comparable to those of the late-L objects in the field. The $\mathrm{K}_{\mathrm{I}}$ absorption is strong and defines the pseudo-continuum of the optical energy distribution (Pavlenko, Zapatero Osorio \& Rebolo 2000; Allard et al. 2001). Although metallic hydrides are clearly present, molecular absorption due to oxides ( $\mathrm{TiO}$ and $\mathrm{VO}$ ) is not obvious, except for a weak feature at 8400-8600 (flat flux) that might be caused by TiO. This adds support to our spectral classification (VO and $\mathrm{TiO}$ have vanished by mid-L types at the typical atmospheric gravities of the field; Kirkpatrick et al. 1999; Martín et al. 1999).

A closer inspection of $\mathrm{FeH}$ features in Fig. 3 reveals that they are slightly stronger in the spectra of the Pleiades objects than in the older, field L6-L7 standards. Furthermore, Calar 21 and 22 present stronger FeH absorption at $9896 \AA$ than the young J1256-1257b (see Fig. 1). This object's age is kinematically estimated at about $300 \mathrm{Myr}$ (Zapatero Osorio et al., in preparation), i.e. older than the Pleiades, which is consistent with the lithium depletion observed in the primary binary brown dwarf of the system (Gauza et al. 2015) and with the age estimate of Rich et al. (2016). Under the assumption that all sources shown in Figs 1 and 3 have identical metallicity, FeH absorption at optical wavelengths appears to be intense in late- $\mathrm{L}$ Pleiades objects. This behaviour is opposite to the one described by McGovern et al. (2004) and Martin et al. (2017): low-gravity dwarfs show weaker hydride absorption. However, these authors focused on the $J$-band spectra of late-M and early-L dwarfs. Lodieu et al. (2017) show spectra of Upper Scorpius candidate members spanning the spectral range L0-L7. FeH is also seen in weak absorption with respect to the high-gravity dwarfs of similar classification. If not due to gravity (atmospheric pressure), other explanations to account for the distinct $\mathrm{FeH}$ intensities are that the Pleiades cluster has different metallicity from the comparison objects, poor $\mathrm{S} / \mathrm{N}$ of our data and bad telluric correction, and/or J1256-1257b and Calar 21 and 22 have distinct spectral types $(\mathrm{FeH}$ becomes weaker with decreasing temperature). The former scenario is less likely, since Pleiades F- and G-type stars have nearly solar Fe abundance (Soderblom et al. 2009; Takeda, Hashimoto \& Honda 2017). The low S/N and unequal classifications are more plausible: J1215-1257b has a $K$-band absolute magnitude $\approx 1.5$ mag fainter than those of Calar 21 and 22 (see Section 3.3). The spectral classification of very faint (low luminosity), dusty dwarfs is not solidly defined yet (Allers \& Liu 2013; Faherty et al. 2016; Liu et al. 2016). By studying a sequence of faint and red sources that have the same age, metallicity and distance but different magnitudes, i.e. members of a star cluster, one can establish the sequence of spectral types in a consistent manner.

There is a tentative detection of neutral cesium at $8521 \AA$ in the merged spectrum of Fig. 3. It is the strongest Cs I atomic line at visible wavelengths. We measured a pseudo-equivalent width (pEW) of $8.6 \pm 0.5 \AA$ by adopting two pseudo-continuum points at 8500 and $8545 \AA$ around the line centre. This value is compatible with the high measurements of field late-L dwarfs reported by Kirkpatrick et al. (1999, 2000), Burgasser et al. (2003), Lodieu et al. (2015), Manjavacas et al. (2016), and references therein. Alkali lines are very sensitive to both temperature and gravity. At low-gravity atmospheres and cool temperatures down to $\approx 1000 \mathrm{~K}$, we would expect weaker atomic lines. Our Cs I $\lambda 8521 \AA \mathrm{pEW}$ may be affected by the very low-spectral resolution of our data and the poor $\mathrm{S} / \mathrm{N}$. We imposed an upper limit of $3.5 \AA$ on Cs I $\lambda 8943 \AA$, and $6 \AA$ on the pEW of Rb i $\lambda 7948 \AA$. This is slightly below the actual pEWs corresponding to field L7 dwarfs, which is consistent with low-pressure atmospheres.

Fig. 7 displays various L-type optical spectra of the very young Upper Scorpius association (UpSco, 5-10 Myr; Preibisch et al. 2002; Song, Zuckerman \& Bessell 2012; Pecaut, Mamajek \& Bubar 2012; Pecaut \& Mamajek 2016) obtained with the same instrumental setup as our data. Each spectrum was fabricated by averaging data of two different UpSco members with the same spectral classification that were recently published by Lodieu et al. (2017). We adopted the 'near-infrared' spectral classification scheme proposed by these authors. The global morphology of the Pleiades spectrum appears very similar to UpSco L7 dwarfs in Fig. 7. FeH $\lambda 8692 \AA$ absorption intensity is slightly stronger in the Pleiades. At earlier spectral types, and as shown in Fig. 7, oxides (TiO and VO) are still present in the optical spectra of very young dwarfs. To study the dependence of spectroscopic features on gravity at late- $\mathrm{L}$ types, data obtained to a higher $\mathrm{S} / \mathrm{N}$ are required. 


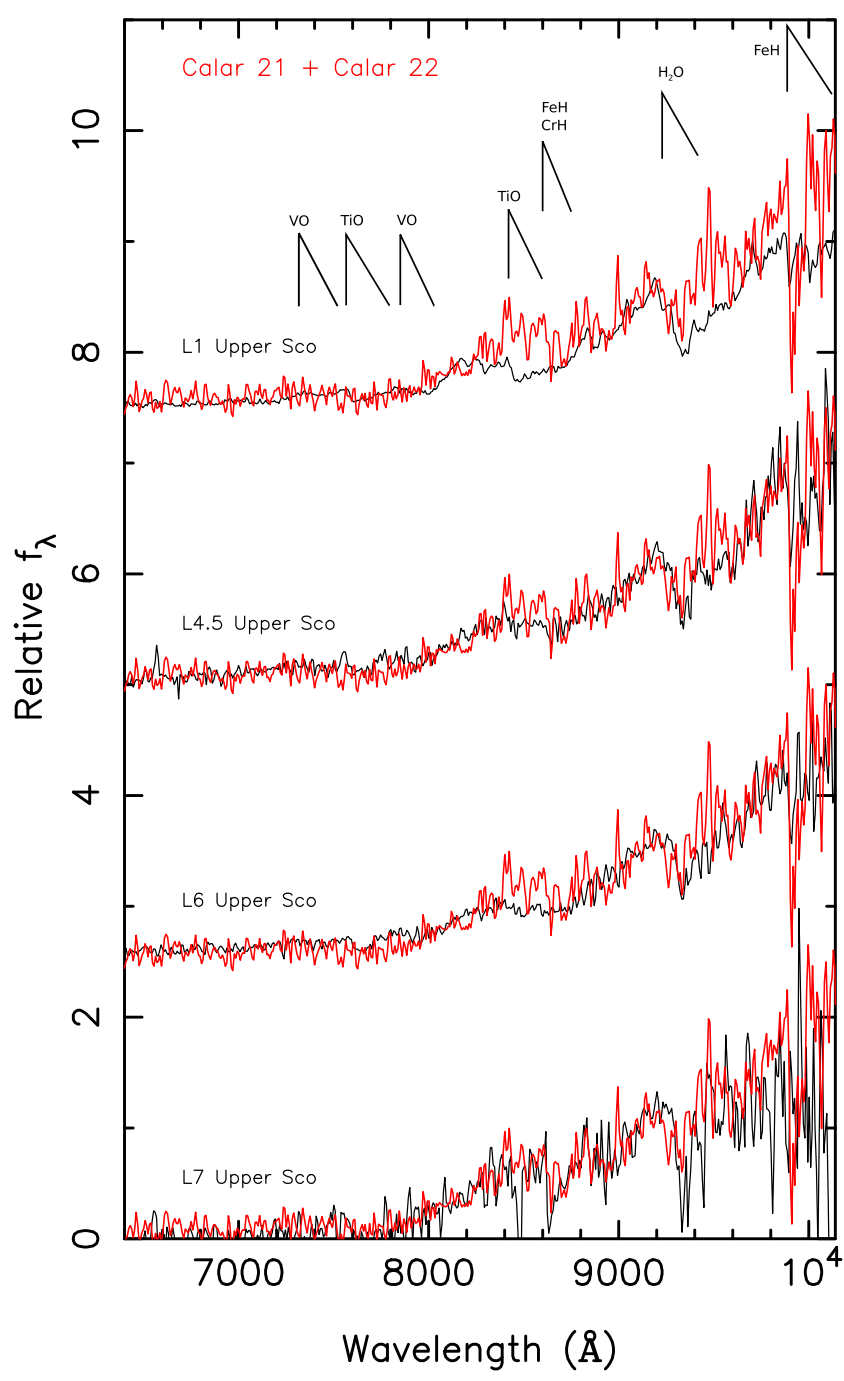

Figure 7. The OSIRIS merged spectrum of Calar 21 and 22 (red) is compared to L-type spectra of Upper Scorpius members (black). All data were obtained with the same instrument, are normalized to unity at $\approx 9100$ $9150 \AA$, and are vertically shifted by a constant for clarity. Some molecular features are indicated after Kirkpatrick et al. (1999).

\subsection{The Pleiades sequence}

Fig. 8 depicts the sequence of Pleiades members contrasted with that of the AB Doradus moving group, which has an age (150 Myr; Bell, Mamajek \& Naylor 2015) very close to the Pleiades. Actually, some authors argue that the Pleiades star cluster and $\mathrm{AB}$ Doradus moving group are likely coeval and share a common origin (e.g. Ortega et al. 2007, and references therein). Only confirmed AB Doradus members with trigonometric distances are shown (see Faherty et al. 2016; Liu et al. 2016 for details). The companion 2MASS J22362452+4751425b (J2236+4751b) to a late-K dwarf likely belonging to the AB Doradus moving group (Bowler et al. 2017) is also included in the diagram, although it has no parallax available to date. Bowler et al. (2017) discuss that J2236+4751b may define the 'elbow' of the AB Doradus photometric sequence separating L dwarfs from the cooler T dwarfs. All AB Doradus candidate members have been taken to the Pleiades distance for a proper comparison. As illustrated in Fig. 8, both Pleiades and AB Doradus sequences of $\mathrm{M}$ and $\mathrm{L}$ dwarfs nicely overlap, including the reddest $J-K$ colours. The exact location of the L/T transition ('elbow') in

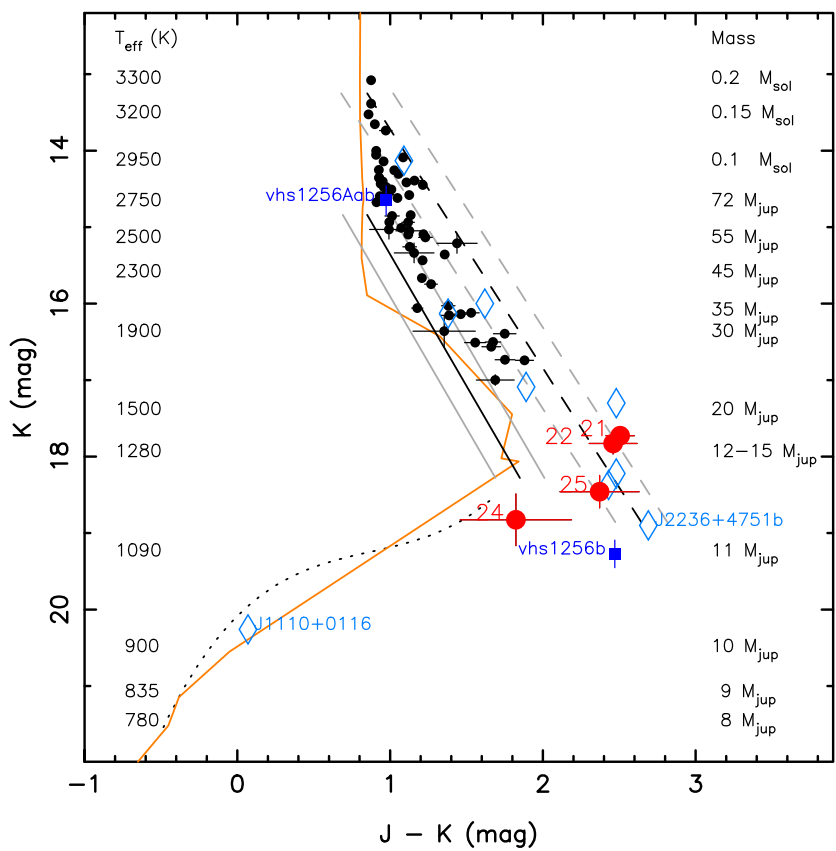

Figure 8. Colour-magnitude diagram of Pleiades members (dots). Red dots stand for the Calar sources (labelled with their identification number) with high-cluster membership probability (this excludes Calar 26). Black dots stand for previously known mid-M through mid-L Pleiades members (Bihain et al. 2010; Zapatero Osorio et al. 2014a). L- and T-type members of the 150-Myr AB Doradus moving group that have trigonometric distances (see Faherty et al. 2016; Liu et al. 2016) are plotted as open blue symbols: the L7 dwarf J2236+4751b (Bowler et al. 2017) in the only AB Dor candidate member that has no parallax to date. The system J1256-1257 (Gauza et al. 2015), which has a likely age in the interval $150-300 \mathrm{Myr}$, is shown with solid blue squares. The black solid and dotted lines show the fits for field, high-gravity L- and T-type dwarfs obtained by Liu et al. (2016) and Filippazzo et al. (2015), respectively. The sequence of low-gravity L dwarfs defined by Liu et al. (2016) is illustrated with a black dashed line. The grey solid and dashed lines show the $1 \sigma$ scatter about their corresponding linear fits. The BT-Settl solar metallicity, 120-Myr isochrone is shown with a brown solid line. Masses and effective temperatures predicted by this evolutionary model are given on both sides of the diagram. All AB Dor members, J1256-1257, the field low- and high-gravity sequences, and the isochrone were taken to the distance of the Pleiades cluster.

the Pleiades is still pending the spectroscopic confirmation of the presence of methane in Calar 24 (see above). Furthermore, these observations would be helpful to constrain the theoretical predictions of Marley et al. (2012). These authors provided a toy model for the definition of the $\mathrm{L} / \mathrm{T}$ transition where the effective temperature $\left(T_{\text {eff }}\right)$ range of the transition is made gravity dependent. The toy model was 'calibrated' against the observations of high-gravity dwarfs and the planets around HR 8799 (Marois et al. 2008, 2010). According to Marley et al. (2012), the 'elbow' of the Pleiades sequence is expected at $900-1000 \mathrm{~K}$ or $10 M_{\text {Jup }}$. This is $\approx 1.5$ mag fainter in $K$ band than the position of Calar 24 or $\mathrm{J} 2236+4751$ b in Fig. 8 . Rajan et al. (2017) have adopted these models as one possibility to explain the red SED of the young T dwarf 51 Eridani b (Macintosh et al. 2015), which orbits 51 Eri A, an F0IV star that is part of the $\beta$ Pictoris moving group.

The Pleiades sequence is also compared to the tracks delineated by high-gravity M, L, and T dwarfs in Fig. 8. The mean location and dispersion of field M6-L8 sources is adopted from Liu et al. (2016); as for the field T dwarf sequence, we adopted Filippazzo et al. (2015). All photometry is taken to the distance of the Pleiades 
Table 3. Absolute magnitudes for L7 dwarfs of different gravity.

\begin{tabular}{lccccl}
\hline SpT & $\begin{array}{c}M(Z) \\
(\mathrm{mag})\end{array}$ & $\begin{array}{c}M(J) \\
(\mathrm{mag})\end{array}$ & $\begin{array}{c}M(H) \\
(\mathrm{mag})\end{array}$ & $\begin{array}{c}M(K) \\
(\mathrm{mag})\end{array}$ & Reference \\
\hline L7 Pleiades & $\approx 17.32$ & $\approx 14.62$ & $\approx 13.20$ & $\approx 12.12$ & This paper \\
L7 VL-G & & $15.90 \pm 0.72$ & $14.54 \pm 0.64$ & $13.30 \pm 0.56$ & Liu et al. (2016) \\
L7 INT-G & & $15.07 \pm 0.50$ & $13.61 \pm 0.43$ & $12.59 \pm 0.39$ & Liu et al. (2016) \\
L7 field & & $14.32 \pm 0.37$ & $13.43 \pm 0.33$ & $12.68 \pm 0.31$ & Liu et al. (2016) \\
L7 field & & $14.46 \pm 0.40$ & $13.35 \pm 0.39$ & $12.62 \pm 0.54$ & Faherty et al. (2016) \\
L7 field & $16.72 \pm 0.52$ & & & & Hewett et al. (2006) \\
\hline
\end{tabular}

cluster. It becomes apparent from the figure that the Pleiades sequence of $\mathrm{M}$, early-L, and mid-L dwarfs is overluminous in the $K$ versus $J-K$ diagram with respect to the field by about $0.5-1 \mathrm{mag}(K)$, as expected for its youth: although at a slower pace than at much younger ages, Pleiades brown dwarfs and planetary-mass objects are still collapsing under their own gravity (Burrows et al. 1993; Baraffe et al. 2002; Saumon \& Marley 2008). As an example, dwarfs with masses of 13 and $50 M_{\text {Jup }}$ have sizes larger than Jupiter at $120 \mathrm{Myr}$ and will have their radii reduced by $\approx 27$ per cent and $\approx 65$ per cent, respectively, between the age of the Pleiades and 5 Gyr.

The faintest and reddest Pleiades members (e.g. Calar 21, 22, and 25$)$ are significantly redder and slightly brighter $(\sim 0-0.5 \mathrm{mag})$ than their field, high-gravity late-L counterparts in the $K$ band (Fig. 8, Table 3). Since one possible explanation for their reddish appearance lies on the presence of very thick clouds of dust, nonequilibrium chemistry, and rapid vertical mixing in the atmospheres (Barman et al. 2011; Madhusudhan, Burrows \& Currie 2011; Skemer et al. 2014, and references therein), it may be feasible that absorption/scattering of condensates is occurring even at long wavelengths, thus yielding 'obscured' $Z, J, H$, and $K$ magnitudes: the shorter the wavelength, the more affected by dust absorption. This would contribute to explain the apparent lack of brightness at these wavelengths with respect to the field. This fact was already pointed out by Marocco et al. (2014), who stated that the diversity in near-infrared colours and spectra seen in late L dwarfs could be due to differences in the optical thickness of the dust cloud deck. This same argument is also employed to account for the observed photometric modulations in ultracool dwarfs (e.g. Yang et al. 2015; Lew et al. 2016, and references therein). For other theoretical interpretations of the very red colours of L dwarfs, see Tremblin et al. (2016).

In Fig. 8, we also included the 'very low gravity' sequence of M6L7 dwarfs defined by Liu et al. (2016, their fig. 14). These authors used the criteria of Allers \& Liu (2013) for classifying the gravity of the near-infrared spectra of young sources in the field and in stellar moving groups. The 'very low gravity' flag roughly corresponds to the optical classification of $\gamma$ (Cruz, Kirkpatrick \& Burgasser 2009) and to ages of $\leq 30 \mathrm{Myr}$ (i.e. $\geq 4$ times younger than the Pleiades). Whereas the very low-gravity sequence runs above the Pleiades between mid-M and mid-L dwarfs in Fig. 8, which is consistent with two distinct ages, we caution that both sequences steadily converge at the late- $\mathrm{L}$ types (or faintest magnitudes). As discussed by Bihain et al. (2006) and Luhman (2012), this feature might be a natural consequence of the early evolution of brown dwarfs and planetary-mass objects: below $\sim 1500 \mathrm{~K}$ all low mass objects are predicted to have similar radii, even the younger ones (Burrows et al. 1997; Chabrier et al. 2000a). However, we remark that atmospheric gravity is not as similar. For instance, a $1300 \mathrm{~K}$ dwarf has a surface gravity of $\log g=4.45\left[\mathrm{~cm} \mathrm{~s}^{-2}\right]$ at the age of $120 \mathrm{Myr}$, $4.06 \mathrm{dex}$ at $20 \mathrm{Myr}$, and $5.10 \mathrm{dex}$ at $1 \mathrm{Gyr}$ (Chabrier et al. 2000a).
Between the youngest and oldest ages, there is a difference of one order of magnitude in the surface gravity for a given temperature. Between 20 and $120 \mathrm{Myr}$, the gravity parameter differs by a factor of 2.5. Yet, this induces noticeable changes in ultracool spectra according to theoretical model atmospheres (e.g. Allard et al. 2001; Marley et al. 2012; see also Section 4.3). Calar 21, 22, and 25 (120 Mr), together with the AB Doradus members (150 Myr) with similar absolute magnitude and colours, and J1256-1257b (150$300 \mathrm{Myr}$ ), strongly demonstrate that intermediate-age late-L dwarfs can posses extreme atmospheric properties typically attributed to much younger objects (e.g. 2MASS J1207334-393254b, Chauvin et al. 2004; PSO J318.5338-22.8603, Liu et al. 2013). See also the discussion by Bowler et al. (2017). We caution that extremely red colours of L dwarfs are not a direct evidence of ages younger than $\approx 100$ Myr.

In the Pleiades, L7 type dwarfs have $Z \approx 22.95, J \approx 20.25$, $H \approx 18.83$, and $K \approx 17.75 \mathrm{mag}$, corresponding to the absolute magnitudes at the age of the cluster (120 Myr) listed in Table 3. In the table, and for comparison purposes, we also included the ZJHK absolute magnitudes representing the high-gravity dwarfs of related classification provided by Hewett et al. (2006, table 10), Liu et al. (2016, table 10), and Faherty et al. (2016, table 19). These values stand for L7 dwarfs that typically have an older age than the Pleiades, and therefore, higher gravity atmospheres. All ZJHK data are in the Mauna Kea Observatories (MKO) photometric system for a direct comparison (Simons \& Tokunaga 2002; Tokunaga, Simons \& Vacca 2002). The 2MASS JHK magnitudes of Faherty et al. (2016) were transformed into the MKO system using the equations of Stephens \& Leggett (2004). We used the mean $Y$ magnitudes of Liu et al. (2016) and the $Z-Y$ colours of Hewett et al. (2006) to fill the $Z$ datum of field L7 dwarfs in Table 3. Pleiades lateL dwarfs are dimmer by $\approx 0.6(Z)$ and $\approx 0.25(J)$ mag than their field spectral counterparts, but are brighter in $H$ and $K$ by similar amounts, a signpost of their extremely red colours. This confirms the 'pivotal' nature of the SEDs of young red L-type objects mentioned by Filippazzo et al. (2015). Since one expects that young, inflated dwarfs are overluminous at all wavelengths with respect to the field, this supports evidence of significant scattering of light in dusty extended ultracool atmospheres.

\subsection{Spectral energy distribution}

We built the SED of Calar 21, 22, and 25 from optical to infrared wavelengths by combining data presented here with data from the discovery paper and Zapatero Osorio et al. (2014b). The goal is a better characterization of the least massive population of the Pleiades. The $Z$ and $H K$ photometry was used to flux calibrate the optical and near-infrared spectra of Calar 21 and 22 by integrating over the spectra using the UKIDSS filters bandpasses. Fig. 9 illustrates the SEDs of Calar 21 and 22 together with the data of the L7 dwarf 

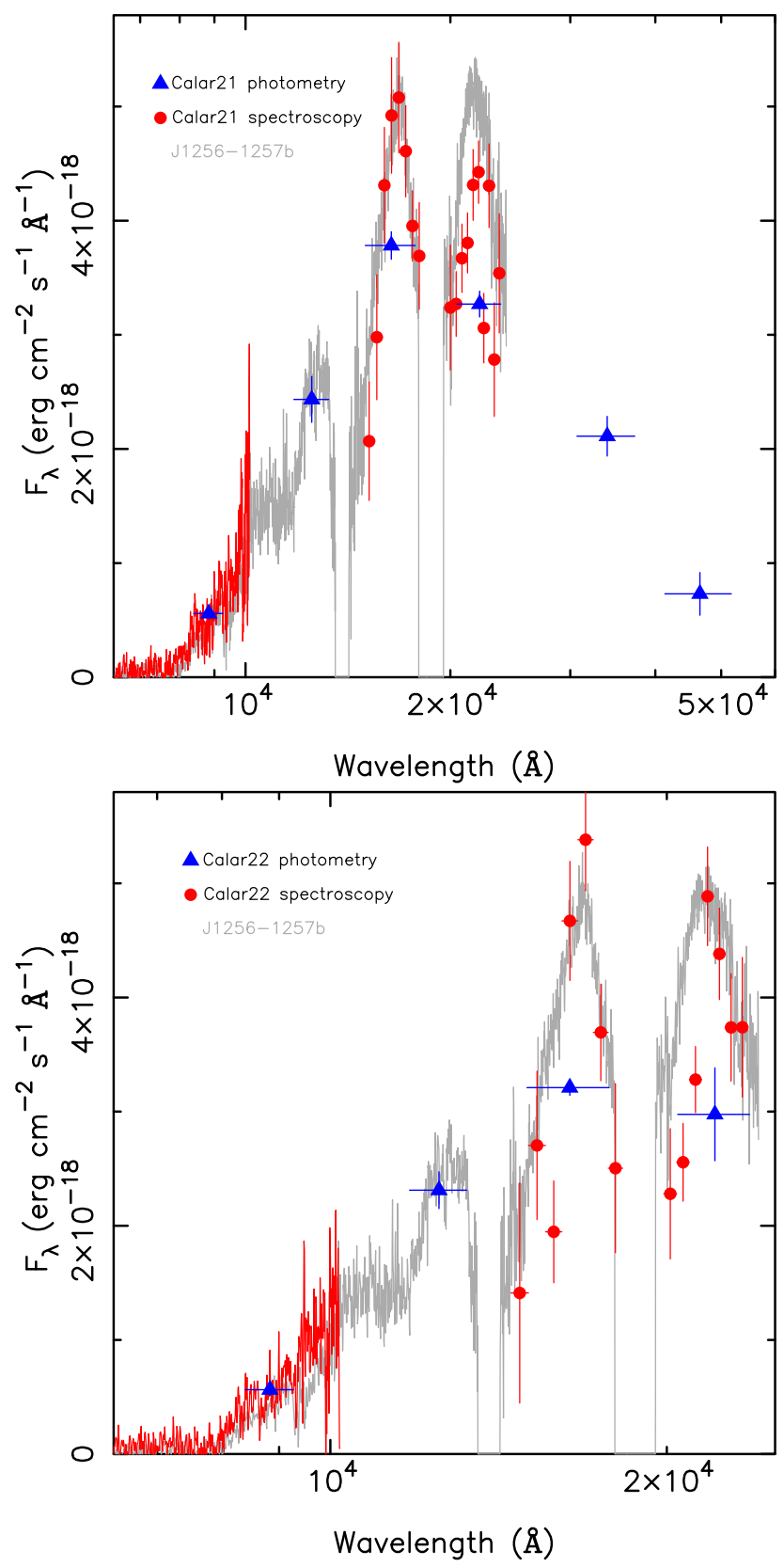

Figure 9. Observed spectrophotometric energy distribution of Calar 21 (top) and 22 (bottom) using optical, near- and mid-infrared data. The optical (this paper) and near-infrared spectra (Zapatero Osorio et al. 2014b) are shown in red colour, while the photometry is plotted as blue solid triangles. For comparison purposes, the spectrum of J1256-1257b (Gauza et al. 2015) is shown with a grey line; it is normalized to the total $J$-band flux of Calar 21 and 22. The horizontal error bars associated with the photometry indicate the passband of the filters. The $x$-axis (wavelength) is shown in logarithmic scale.

J1256-1257b (Gauza et al. 2015), which were normalized to the $J$-band fluxes of the Calar sources. The spectral shapes of Calar 21 and 22 closely match the SED of J1256-1257b, except for the $K$ band, where the latter object is overluminous, which is consistent with its redder $J-K$ colour.

The solar metallicity BT-Settl model atmospheres (Baraffe et al. 2015; see also Manjavacas et al. 2016) were compared to the observed SEDs to assess the most likely $T_{\text {eff }}$ and gravity $(\log g)$ parameters. These models self-consistently calculate the thermal structure and equilibrium chemistry for cloud-bearing atmospheres with solar abundance (Caffau et al. 2011) at different resolutions in a wide range of wavelengths. The models also account for the formation and gravitational settling of dust grains at low temperatures. We identified the best spectral fits by visual inspection of the comparisons, which included theoretical spectra covering the following intervals: $1400-1100 \mathrm{~K}$ and $2.0-5.0\left[\mathrm{~cm} \mathrm{~s}^{-2}\right]$ with steps of $100 \mathrm{~K}$ and 0.5 dex in $T_{\text {eff }}$ and $\log g$, respectively. Hereafter, models are denoted by the doublets $T_{\text {eff }} / \log g$.

We found that Calar 21 and 22 are reasonably reproduced by the 1300/2.0 BT-Settl model, while Calar 25's photometric SED is well matched by the 1200/2.0 model. Results are shown in Fig. 10. Regarding Calar 25, all photometric data points are reproduced to better than $1 \sigma$ the quoted uncertainties. Of the explored grid of models, the 1300/2.0 synthetic spectrum displays the reddest colours, i.e. the steepest rising slope between visible wavelengths and the $K$ band. At warmer and cooler temperatures, the $J-K$ colours are bluer. The BT-Settl models point to that $\sim 1300 \mathrm{~K}$ is the temperature at which the settlement of the grains commences for low-gravity atmospheres. The derived $T_{\text {eff }} \mathrm{s}$ for Calar 21, 22, and 25 are consistent with those estimated from evolutionary models (Section 4.5). We assigned an error of $\pm 100 \mathrm{~K}$ to the temperatures estimated from the spectral fitting technique.

However, the spectral fitting analysis yielded a surprisingly low surface gravity that is about two orders of magnitude lower than predicted by the 120-Myr isochrone (Baraffe et al. 2015) depicted in Figs 5 and 8 (see Section 4.2). This low-gravity result is driven by the very red colours of the Calar sources. Very low gravities have an effect on the objects' size and mass: for a given mass, the radius increases by a factor or 10 , or the mass decreases by a factor of 100 for a fixed size. No evolutionary model available to us predicts such a low gravity for any substellar object with a mass in the interval $0.5-72 M_{\text {Jup }}$ at the age of the Pleiades cluster. The enormous disagreement in the gravity derivations indicates that some physics of substellar evolution is unaccounted for, or that the structure of the dusty atmospheres is poorly determined by the theory, or that the condensate chemistry and contribution of dust to the atmospheres are not properly treated in the computations, or all together. Note that the measured radii of old brown dwarfs by the techniques of transit duration and orbital velocity of doublelined eclipsing binaries (see Filippazzo et al. 2015 for a compilation of data) agree with the evolutionary models for typical ages of Gyr (e.g. Littlefair et al. 2014).

Yet, the BT-Settl model atmospheres can be used to determine the trend of various atomic and molecular features with gravity and temperature in qualitatively terms. For a given low temperature, the lower the gravity, the steepest the spectral slope from visible through $\sim 2 \mu \mathrm{m}$. In addition, methane absorption at 2.2 and $\sim 3.4 \mu \mathrm{m}$, which is present in high-gravity $1200-\mathrm{K}$ field dwarfs (early-T types, e.g. Vrba et al. 2004), is clearly sensitive to gravity: methane absorption considerably reduces at low gravity. Oxides have an opposite behaviour: they become stronger with lower gravity. A detailed inspection of Fig. 10 reveals that some optical and near-infrared $\mathrm{TiO}$ and $\mathrm{VO}$ absorption occurs at low atmospheric pressures and temperatures, whereas these molecules disappear at high gravities for the same low temperatures. Since the M/L/T types are defined based on the presence and disappearance of certain molecules from the observed spectra (e.g. $\mathrm{TiO}, \mathrm{VO}, \mathrm{CrH}, \mathrm{FeH}, \mathrm{CO}, \mathrm{CH}_{4}$ ), this quite likely affects the establishment of a spectral type definiton for very low-gravity dwarfs (see Lodieu et al. 2017; Zapatero Osorio et al. 2017). 

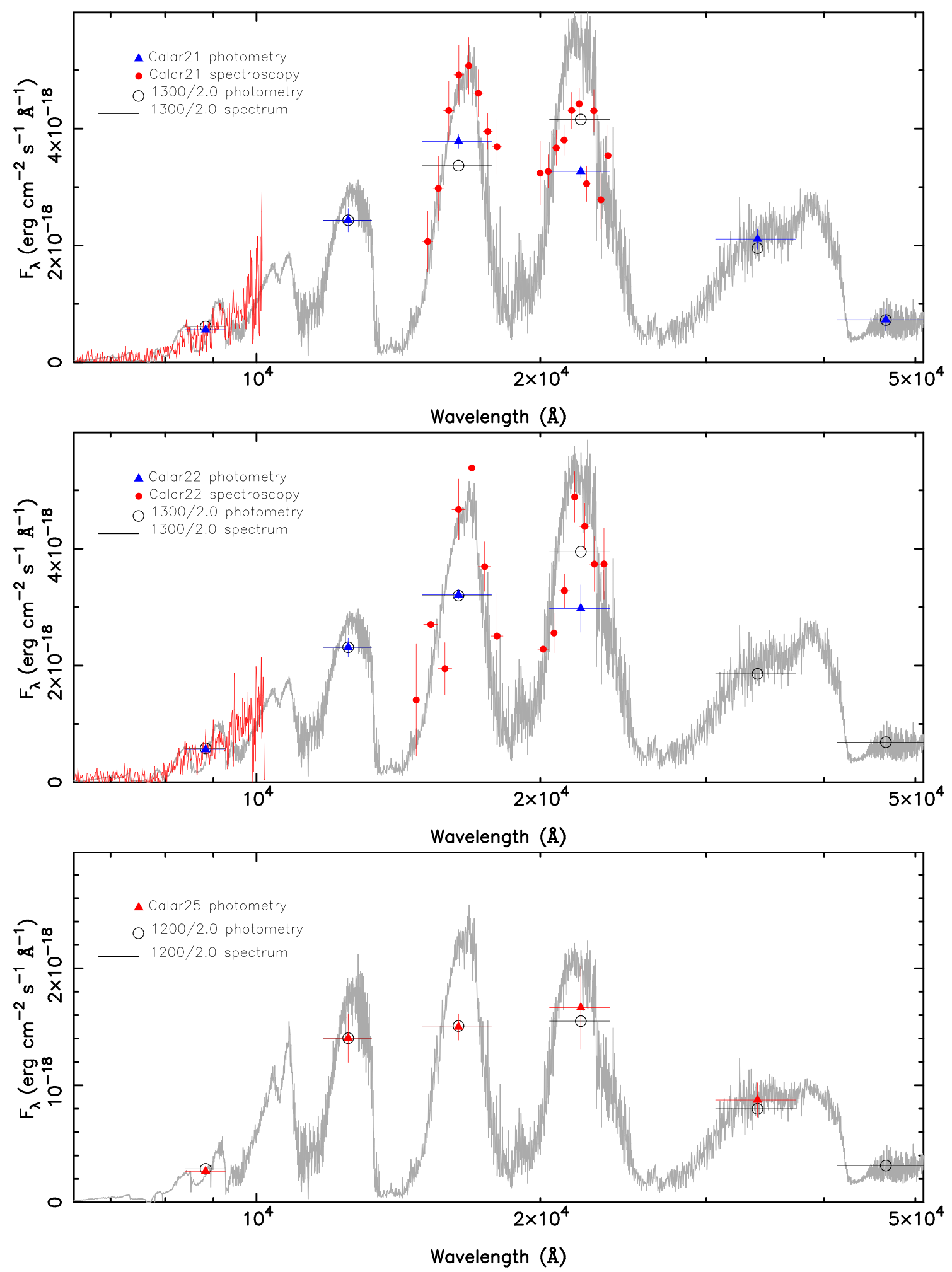

Figure 10. Observed spectrophotometric energy distribution of Calar 21 (top), Calar 22 (middle), and Calar 25 (bottom). Synthetic BT-Settl spectra are depicted with grey solid lines. Theoretical photometry as integrated from the displayed models are plotted as open black circles. The models are normalized to the $J$-band fluxes of each Calar source. The horizontal error bars associated with the photometry indicate the passband of the filters (from blue to red wavelengths: $Z, J, H, K, W 1$, and $W 2$ ). The $x$-axis (wavelength) is shown in logarithmic scale. The models shown correspond to solar metallicity, $T_{\text {eff }}=1300$ and $1200 \mathrm{~K}$, and $\log g=2.0$ dex. 
Table 4. Mass, luminosity, $T_{\text {eff }}$, and bolometric correction for three Pleiades low mass members.

\begin{tabular}{lcccccc}
\hline Object & $\begin{array}{c}\text { Mass } \\
\left(M_{\text {Jup }}\right)\end{array}$ & $\begin{array}{c}\log L / \mathrm{L} \odot \\
(\mathrm{dex})\end{array}$ & $\begin{array}{c}T_{\text {eff }} \\
(\mathrm{K})\end{array}$ & $\begin{array}{c}\mathrm{BC}(J) \\
(\mathrm{mag})\end{array}$ & $\begin{array}{c}\mathrm{BC}(K) \\
(\mathrm{mag})\end{array}$ & $\begin{array}{c}\mathrm{BC}(W 1) \\
(\mathrm{mag})\end{array}$ \\
\hline Calar 21 & $15_{-3}^{+5}$ & $-4.29 \pm 0.10$ & $1350_{-80}^{+100}$ & $0.86 \pm 0.15$ & $3.37 \pm 0.10$ & $4.62 \pm 0.15$ \\
Calar 22 & $15_{-3}^{+5}$ & $-4.33 \pm 0.10$ & $1350_{-80}^{+180}$ & $0.89 \pm 0.15$ & $3.36 \pm 0.20$ & - \\
Calar 25 & $11.5 \pm 0.5$ & $-4.66 \pm 0.10$ & $1150_{-100}^{+50}$ & $1.18 \pm 0.20$ & $3.55 \pm 0.25$ & $4.57 \pm 0.20$ \\
\hline
\end{tabular}

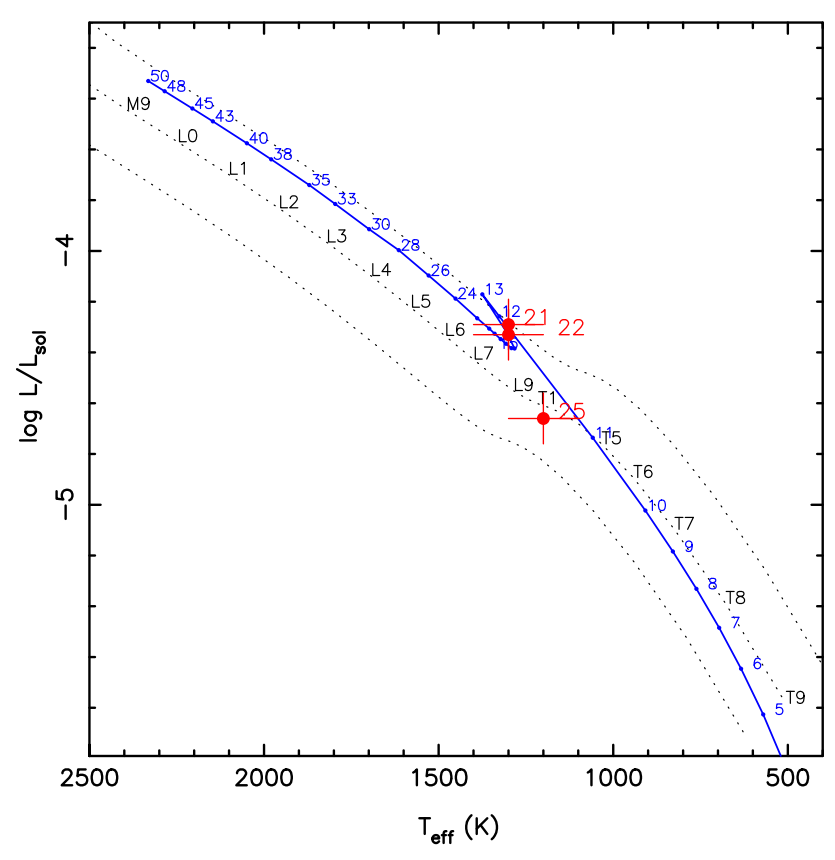

Figure 11. Hertzsprung-Russell diagram of Calar 21, 22, and 25 (red dots labelled wih the Calar numbers). The field sequence of mid-M through T9 dwarfs defined by Filippazzo et al. (2015) is indicated with a dotted black line labelled with spectral types. Dotted lines above and below stand for the dispersion observed in the field. The solar metallicity, 120-Myr evolutionary model of Saumon \& Marley (2008) is depicted with a solid blue line. Labels in blue correspond to the mass given by the model in Jovian units $\left(1 \mathrm{M}_{\odot} \approx\right.$ $\left.1000 M_{\text {Jup }}\right)$. The kink at about $1300 \mathrm{~K}$ correspond to the deuterium burning phase at the age of the Pleiades.

\subsection{Luminosities and bolometric corrections}

We estimated the bolometric luminosity for Calar 21, 22, and 25 using the observed spectra and photometry together with the BTSettl models that provide the best fits to the data as a bolometric correction for short and long wavelengths not covered by the observations. We integrated the extended SEDs over the wavelength interval 3000-10 $10^{6} \AA$ using the simple trapezoidal rule and applied $m_{\text {bol }}=-2.5 \log f_{\text {bol }}-18.9974$, where $f_{\text {bol }}$ is in units of $\mathrm{W} \mathrm{m}^{-2}$, to obtain the apparent bolometric magnitudes. They were then converted into absolute bolometric magnitudes using the distance to the cluster of $133.5 \mathrm{pc}$ (Galli et al. 2017, and references therein). The solar bolometric magnitude of $M_{\mathrm{bol}}=+4.74$ mag was employed to obtain the luminosities of the Calar sources. Results are provided in Table 4. Uncertainties in $\log L / \mathrm{L}_{\odot}$ were derived from the photometric error bars and the distance error. At the age of the Pleiades, these luminosities correspond to the masses also listed in Table 4 and discussed in Section 4.5.

Fig. 11 depicts the Hertzsprung-Russell diagram for Calar 21, 22, and 25 in comparison with the field sequence of ultracool dwarfs defined by Filippazzo et al. (2015). Despite being dimmer in $Z$ and $J$ bands, these Calar objects maintain about the same bolometric luminosity as field dwarfs of similar temperature. Particularly, Calar 25 has a bolometric luminosity that is undistinguishable from the field. This implies that objects with masses around the deuterium burning-mass limit cool down from the Pleiades age up to Gyr following the track delineated by field late-L and T dwarfs.

The $J$ - and $K$-band bolometric corrections (BCs) of Calar 21, 22, and 25 (Table 4) were obtained from the equation $\mathrm{BC}(X)=m_{\mathrm{bol}}-X$, where $X$ stands for the apparent magnitude in each observing filter. Calar 21 and 22 have BCs that agree with those measured by Filippazzo et al. (2015) for young dwarfs: $\mathrm{BC}(K)$ is consistent with high-gravity dwarfs of related types while $\mathrm{BC}(J)$ appears smaller by about 1 mag with respect to the field (we employed the following references for field BCs: Dahn et al. 2002; Golimowski et al. 2004; Vrba et al. 2004; Looper et al. 2008). This highly contrasts with the results of Todorov, Luhman \& McLeod (2010) and Lodieu et al. (2017), who found that $\mathrm{BC}(K)$ is larger for the very young Taurus and Upper Scorpius L-type dwarfs. This may be attributable to the different ages $(\sim 1$ and $\sim 10 \mathrm{Myr}$ versus $120 \mathrm{Myr}$ ) of the samples and to the fact that none of the Taurus and Upper Scorpius sources in those works appear to be as red as our targets. The BCs of Calar 25, whose spectral type is expected to be equal or cooler than Calar 21 and 22, however, show different properties: $\mathrm{BC}(K)$ is larger than the field (in better agreement with Todorov et al. 2010 and Lodieu et al. 2017 findings) while $\mathrm{BC}(\mathrm{J})$ lies between the values corresponding to young late-L dwarfs of Filippazzo et al. (2015) and those of the field. These results confirm that BCs of L dwarfs are a function of other parameters than temperature alone: surface gravity, dust concentration, etc.

\subsection{Masses}

Figs 5 and 8 also depict the BT-Settl 120-Myr isochrone computed for solar metallicity by Allard (2014) and Baraffe et al. (2015). The evolutionary model makes predictions on the behaviour of the optical and near-infrared colours as a function of mass (or $T_{\text {eff }}$ or luminosity), some of which can be tested against the observed sequence of Pleiades low-mass members. The obvious visual result from Figs 5 and 8 is that the isochrone lacks quantitative accuracy while reproducing the increasing reddening of the cluster sequence from the $\mathrm{M}$ through the $\mathrm{L}$ types in a qualitative manner. For example, the $Z-K$ and $J-K$ colours appear to saturate at values of $\sim 4.2$ and $1.8 \mathrm{mag}$ according to the theory, but Calar 21, 22, and 25 have redder indices by about 1 mag in $Z-K$ (top panel of Fig. 5) and about 0.5 mag in $J-K$ (Fig. 8); and the $Z-J$ colour bends to blue and then to red values, but the theoretical $J$-band magnitude at which $Z-J$ turns back to red is off by about 0.5 mag with respect to the observed sequence (bottom panel of Fig. 5). In the magnitude ranges shown in the figures, the $Z-K$ index remains 'saturated' down to very faint magnitudes, whereas the $J-K$ colour turns to blue indices caused by the onset of methane absorption and/or the settlement of dust particles below the photospheres. This turnover is predicted to occur 
at $K \approx 18$ mag at the age and distance of the Pleiades by the BT-Settl model, i.e. a place in between the positions of Calar 22 and Calar 25.

To determine masses, we fixed the age and metallicity of our targets to $120 \mathrm{Myr}$ and solar abundance, respectively. Then, masses were directly obtained from the comparison of the derived bolometric luminosities against the luminosity predictions made by the BT-Settl evolutionary model. This method avoids the uncertainties associated with the derivation of $T_{\text {eff }}$ and with the obtention of magnitudes from computed model atmospheres that do not reliably reproduce the observed spectra. Calar 21 and 22 have a mass of 12-20 $M_{\text {Jup }}$ and define the brown dwarf-planetary mass transition in the Pleiades cluster. The isochrone also delivers $T_{\text {eff }}$ for a given luminosity, which is about $1350 \mathrm{~K}$ in the case of Calar 21 and 22. The fainter and redder source Calar 25 has a cooler $T_{\text {eff }}$ of $\sim 1150 \mathrm{~K}$ and a smaller planetary mass near $11.5 M_{\text {Jup }}$. Table 4 summarizes the results. The errors of the mass and temperature derivations account for the quoted uncertainties in luminosity. For comparison purposes, the masses and $T_{\text {eff }}$ 's provided by the BT-Settl 120-Myr isochrone for different magnitudes and filters are also labelled in Figs 5 and 8. Our luminosity-based mass and temperature derivations agree at the level of $1 \sigma$ with the predictions made for the $K$ band, whereas they deviate significantly from the values inferred from the $J$ band $(J$ band-based masses and temperatures are 30-45 per cent smaller). This is likely explained by the errors introduced by the theory of model atmospheres.

The masses indicated in Table 4 are very similar to those obtained using the evolutionary cooling sequences of Saumon \& Marley (2008). In order to illustrate the comparison of our data with other evolutionary models, we plotted the Saumon \& Marley (2008) $120 \mathrm{Myr}$, solar metallicity isochrone in Fig. 11. We selected the 'hybrid' cloudy/cloudless model, i.e. the computations of an evolution sequence that models the $\mathrm{L} / \mathrm{T}$ transition by increasing the sedimentation parameter as a function of decreasing $T_{\text {eff }}$. The positions of Calar 21, 22, and 25 are reasonably well described by this 'hybrid' model, from which we inferred masses of 12-22 $M_{\text {Jup }}$ for Calar 21 and 22, and 11-12 $M_{\text {Jup }}$ for Calar 25. If Calar 24 were finally confirmed as a Pleiades member, its mass would be estimated at $\approx 11 M_{\text {Jup. }}$. In Fig. 11 , the phase of deuterium burning is revealed by the kink in the isochrone at $\sim 1300 \mathrm{~K}$ or $12-13 M_{\text {Jup }}$ at the age of the cluster.

One intriguing feature in Fig. 11 is the pivotal appearance of the theoretical 120-Myr isochrone with respect to the observed sequence defined by field ultracool dwarfs (Filippazzo et al. 2015): whereas the model is overluminous at warm temperatures, as expected for young ages, it runs below the field sequence for $T_{\text {eff }} \leq$ $1100 \mathrm{~K}$ suggesting that Pleiades very low-mass members might appear underluminous with respect to the field. We caution that this is not the correct interpretation. Evolutionary models do not predict this underluminosity nature: isochrones of older ages are systematically less luminous than younger isochrones for all masses. The Saumon \& Marley (2008) isochrones computed for ages of Gyr do not accurately reproduce the field sequence. This may be caused by the way the average field sequence is obtained (mixing objects of very different ages and metallicities), or by an error in the determination of bolometric luminosities and temperatures in the very low-mass regime, or by physics unaccounted for in the evolutionary models.

Calar 21, 22, and 25 are excellent targets for carrying out the test of deuterium (Béjar, Zapatero Osorio \& Rebolo 1999) that has been proposed to discriminate brown dwarfs and planetary-mass objects. All three objects are coeval and have the same metallicity, and their masses are estimated slightly above and below the deuterium burning mass limit at 12-13 $M_{\text {Jup }}$ (Burrows et al. 1993; Chabrier et al. 2000b; Saumon \& Marley 2008). Calar 25 is currently the coolest, least luminous and least massive object whose membership in the Pleiades is astrometrically and photometrically confirmed using data from optical to infrared wavelengths. Its finding demonstrates that despite their very low mass and current dynamically relaxed state of the cluster (Jameson et al. 2002; Zapatero Osorio et al. 2014a), these objects have not completely escaped the Pleiades.

\section{CONCLUSIONS}

This paper presents the follow-up optical imaging and spectroscopy of the six faintest Pleiades candidate members announced in Zapatero Osorio et al. (2014a): Calar 21-26. All data were acquired with the OSIRIS instrument of the GTC. The targets have $J$-band magnitudes in the interval 20.2-21.2 mag; both Z-band photometry and low-resolution spectroscopy $(R \approx 270,6400-10150$ $\AA)$ were obtained for Calar 21 and $22(J \sim 20.2 \mathrm{mag})$, while the fainter sources were observed in the $Z$ band only (except for Calar 24 , which was also imaged in the $i$ band). From the imaging analysis, we determined that Calar 23 is resolved and that Calar 26 does not have a proper motion compatible with the Pleiades motion. Therefore, these two objects are regarded as likely non-members of the cluster. The remaining four candidates have new proper motion determinations that agree with the values published in the original paper at the $1 \sigma$ level and are fully compatible with the Pleiades motion. Calar 21,22 , and 25 show very red $Z-J, Z-K$, and $J-K$ colours that overlap with the colours of the reddest known $L$ dwarfs in young stellar moving groups and the field. Despite having similar brightness to Calar 25, Calar 24 is not as red, which may suggest that it is a contaminant of the astrometric survey. However, the colours of Calar 24 are compatible with field late-L or early-T dwarfs, and the position of this object in colour-magnitude diagrams coincides with the expected location of the T-type sequence of the Pleiades (according to evolutionary models). On the one hand, we conclude that current data cannot rule out that Calar 24 is an unreddened cluster member. Further, near-infrared spectroscopy is required to confirm the low-gravity nature of this particular object. On the other hand, we also conclude that Calar 25 remains as an astrometric and photometric likely member of the cluster; it is the reddest cluster object at optical-to-infrared wavelengths. Based on their astrometric, photometric, and spectroscopic properties, Calar 21 and 22 are bona-fide Pleiades members. Calar 21, 22, and 25 currently define the least luminous and least massive tail of the Pleiades sequence.

The optical spectra of Calar 21 and 22 were compared to primary L dwarf standards for spectral classification. We determined L6-L7, which is consistent with the classification from near-infrared spectra (Zapatero Osorio et al. 2014b). This agreement between optical and near-infrared spectral types contrasts with the findings in other significantly younger ( $\leq 10 \mathrm{Myr}$ ) clusters like Upper Scorpius (Lodieu et al. 2017) and $\sigma$ Orionis (Zapatero Osorio et al. 2017). These types are expected for Pleiades members with $J \sim 20.2$ mag. At the resolution and quality of the data, there are no strong signatures of $\mathrm{TiO}$ and $\mathrm{VO}$ absorption and the following features were identified: a markedly rising pseudo-continuum towards longer wavelengths, which is consistent with very red colours, potassium resonance doublet and a tentative detection of neutral cesium at $8521 \AA$, and absorption due to ferrum and chromium hydrides. Despite the intermediate-gravity nature of the atmospheres expected for Pleiades low-mass dwarfs (120 Myr), these features show intensities that resemble those of high-gravity dwarfs of similar 
classification. The comparison with BT-Settl model atmospheres (Baraffe et al. 2015) allowed us to derive effective temperatures of $\sim 1300 \mathrm{~K}$ for Calar 21 and 22 , and $\sim 1200 \mathrm{~K}$ for Calar 25 (error bar of $\pm 100 \mathrm{~K}$ ), which are close to those obtained from $120-\mathrm{Myr}$ isochrones $(1350$ and $1150 \mathrm{~K}$, respectively). The bolometric luminosities of Calar 21, 22, and 25 were determined from the integration of their corresponding observed SEDs, which allowed us to measure bolometric corrections at near-infrared wavelengths for low-luminous Pleiades members. These bolometric corrections differ from those of highgravity dwarfs of related classification in the line indicated by Filippazzo et al. (2015) for low-gravity atmospheres. Interestingly, they also differ from the bolometric corrections of much younger L-type sources as measured by Lodieu et al. (2017). From evolutionary models (Chabrier et al. 2000a; Saumon \& Marley 2008), the mass of Calar 21 and 22 is estimated at 12-20 $M_{\text {Jup }}$, while the mass of the least massive Pleiades member, Calar 25, is estimated at 11-12 $M_{\text {Jup }}$, below the deuterium burning mass limit. Calar 25 thus turns out to be an excellent candidate for the search for deuterium using upcoming facilities, like JWST. Calar 21, 22, and 25 indicate that the extreme reddish nature of $\mathrm{L}$ dwarfs is not always associated with ages younger than $\approx 100 \mathrm{Myr}$.

\section{ACKNOWLEDGEMENTS}

We thank the anonymous referee for useful comments and suggestions. Based on observations made with the Gran Telescopio Canarias (GTC), installed at the Spanish Observatorio del Roque de los Muchachos of the Instituto de Astrofísica de Canarias, in the island of La Palma. MRZO, VJSB, and NL acknowledges the financial support from the Spanish Ministry of Economy and Competitivity through the projects AYA2016-79425-C3-2-P and AYA201569350-C3-2-P.

\section{REFERENCES}

Alam S. et al., 2015, ApJS, 219, 12

Allard F., 2014, in Booth M., Matthews B. C., Graham J. R., eds, IAU Symp. 299, Exploring the Formation and Evolution of Planetary Systems. Victoria, Canada, p. 271

Allard F., Hauschildt P. H., Alexander D. R., Tamanai A., Schweitzer A., 2001, ApJ, 556, 357

Allers K. N., Liu M. C., 2013, ApJ, 772, 79

Alves de Oliveira C., Moraux E., Bouvier J., Duchêne G., Bouy H., Maschberger T., Hudelot P., 2013, A\&A, 549, A123

Baraffe I., Chabrier G., Allard F., Hauschildt P. H., 2002, A\&A, 382, 563

Baraffe I., Homeier D., Allard F., Chabrier G., 2015, A\&A, 577, A42

Barman T. S., Macintosh B., Konopacky Q. M., Marois C., 2011, ApJ, 735, L39

Basri G., Marcy G. W., Graham J. R., 1996, ApJ, 458, 600

Béjar V. J. S., Zapatero Osorio M. R., Rebolo R., 1999, ApJ, 521, 671

Bell C. P. M., Mamajek E. E., Naylor T., 2015, MNRAS, 454, 593

Bihain G., Rebolo R., Béjar V. J. S., Caballero J. A., Bailer-Jones C. A. L., Mundt R., Acosta-Pulido J. A., Manchado Torres A., 2006, A\&A, 458, 805

Bihain G., Rebolo R., Zapatero Osorio M. R., Béjar V. J. S., Caballero J. A., 2010, A\&A, 519, A93

Boudreault S., Lodieu N., Deacon N. R., Hambly N. C., 2012, MNRAS, 426, 3419

Bowler B. P. et al., 2017, AJ, 153, 18

Burgasser A. J., Kirkpatrick J. D., Liebert J., Burrows A., 2003, ApJ, 594, 510

Burrows A., Hubbard W. B., Saumon D., Lunine J. I., 1993, ApJ, 406, 158

Burrows A. et al., 1997, ApJ, 491, 856
Caffau E., Ludwig H.-G., Steffen M., Freytag B., Bonifacio P., 2011, Sol. Phys., 268, 255

Casewell S. L., Dobbie P. D., Hodgkin S. T., Moraux E., Jameson R. F., Hambly N. C., Irwin J., Lodieu N., 2007, MNRAS, 378, 1131

Casewell S. L., Dobbie P. D., Hodgkin S. T., Moraux E., Jameson R. F., Hambly N. C., Irwin J., Lodieu N., 2010, MNRAS, 402, 1407

Casewell S. L., Jameson R. F., Burleigh M. R., Dobbie P. D., Roy M., Hodgkin S. T., Moraux E., 2011, MNRAS, 412, 2071

Casewell S. L., Baker D. E. A., Jameson R. F., Hodgkin S. T., Dobbie P. D., Moraux E., 2012, MNRAS, 425, 3112

Casewell S. L., Littlefair S. P., Burleigh M. R., Roy M., 2014, MNRAS, 441,2644

Cepa J. et al., 2000, in Iye M., Moorwood A. F., eds, Proc. SPIEConf. Ser. Vol. 4008, Optical and IR Telescope Instrumentation and Detectors. SPIE, Bellingham, p. 623

Chabrier G., Baraffe I., Allard F., Hauschildt P., 2000a, ApJ, 542, 464

Chabrier G., Baraffe I., Allard F., Hauschildt P., 2000b, ApJ, 542, L119

Chauvin G., Lagrange A.-M., Dumas C., Zuckerman B., Mouillet D., Song I., Beuzit J.-L., Lowrance P., 2004, A\&A, 425, L29

Cruz K. L., Kirkpatrick J. D., Burgasser A. J., 2009, AJ, 137, 3345

Cushing M. C. et al., 2008, ApJ, 678, 1372

Dahn C. C. et al., 2002, AJ, 124, 1170

Dawson P., Scholz A., Ray T. P., 2011, MNRAS, 418, 1231

Delfosse X. et al., 1997, A\&A, 327, L25

Downes J. J. et al., 2014, MNRAS, 444, 1793

Dupuy T. J., Liu M. C., 2012, ApJS, 201, 19

Esplin T. L., Luhman K. L., 2017, AJ, 154, 134

Faherty J. K., Burgasser A. J., Cruz K. L., Shara M. M., Walter F. M., Gelino C. R., 2009, AJ, 137, 1

Faherty J. K., Beletsky Y., Burgasser A. J., Tinney C., Osip D. J., Filippazzo J. C., Simcoe R. A., 2014, ApJ, 790, 90

Faherty J. K. et al., 2016, ApJS, 225, 10

Filippazzo J. C., Rice E. L., Faherty J., Cruz K. L., Van Gordon M. M., Looper D. L., 2015, ApJ, 810, 158

Gagné J., Lafrenière D., Doyon R., Malo L., Artigau É., 2014, ApJ, 783, 121

Galli P. A. B., Moraux E., Bouy H., Bouvier J., Olivares J., Teixeira R., 2017, A\&A, 598, A48

Gauza B., Béjar V. J. S., Pérez-Garrido A., Rosa Zapatero Osorio M., Lodieu N., Rebolo R., Pallé E., Nowak G., 2015, ApJ, 804, 96

Golimowski D. A. et al., 2004, AJ, 127, 3516

Hewett P. C., Warren S. J., Leggett S. K., Hodgkin S. T., 2006, MNRAS, 367,454

Jameson R. F., Dobbie P. D., Hodgkin S. T., Pinfield D. J., 2002, MNRAS, 335,853

Kirkpatrick J. D. et al., 1999, ApJ, 519, 802

Kirkpatrick J. D. et al., 2000, AJ, 120, 447

Kniazev A. Y. et al., 2013, ApJ, 770, 124

Lawrence A. et al., 2007, MNRAS, 379, 1599

Lew B. W. P. et al., 2016, ApJ, 829, L32

Littlefair S. P. et al., 2014, MNRAS, 445, 2106

Liu M. C. et al., 2013, ApJ, 777, L20

Liu M. C., Dupuy T. J., Allers K. N., 2016, ApJ, 833, 96

Lodieu N., 2013, MNRAS, 431, 3222

Lodieu N., Deacon N. R., Hambly N. C., 2012, MNRAS, 422, 1495

Lodieu N., Zapatero Osorio M. R., Rebolo R., Béjar V. J. S., Pavlenko Y., Pérez-Garrido A., 2015, A\&A, 581, A73

Lodieu N., Zapatero Osorio M. R., Béjar V. J. S., Peña Ramírez K., 2017, MNRAS, 473, 2020

Loktin A. V., Beshenov G. V., 2003, Astron. Rep., 47, 6

Looper D. L., Gelino C. R., Burgasser A. J., Kirkpatrick J. D., 2008, ApJ, 685,1183

Lucas P. W. et al., 2013. Presented at the workshop on Exoplanets and Brown Dwarfs: Mind the Gap, 2-5 September. Hertfordshire, UK

Luhman K. L., 2012, ARA\&A, 50, 65

Luhman K. L., 2013, ApJ, 767, L1

Luhman K. L., Esplin T. L., Loutrel N. P., 2016, ApJ, 827, 52 
Luhman K. L., Mamajek E. E., Shukla S. J., Loutrel N. P., 2017, AJ, 153, 46

Macintosh B. et al., 2015, Science, 350, 64

Madhusudhan N., Burrows A., Currie T., 2011, ApJ, 737, 34

Manjavacas E. et al., 2016, MNRAS, 455, 1341

Marley M. S., Saumon D., Cushing M., Ackerman A. S., Fortney J. J., Freedman R., 2012, ApJ, 754, 135

Marocco F. et al., 2014, MNRAS, 439, 372

Marois C., Macintosh B., Barman T., Zuckerman B., Song I., Patience J., Lafrenière D., Doyon R., 2008, Science, 322, 1348

Marois C., Zuckerman B., Konopacky Q. M., Macintosh B., Barman T., 2010, Nature, 468, 1080

Martín E. L., Basri G., Gallegos J. E., Rebolo R., Zapatero-Osorio M. R., Bejar V. J. S., 1998a, ApJ, 499, L61

Martín E. L., Basri G., Zapatero-Osorio M. R., Rebolo R., García López R. J., 1998b, ApJ, 507, L41

Martín E. L., Delfosse X., Basri G., Goldman B., Forveille T., Zapatero Osorio M. R., 1999, AJ, 118, 2466

Martin E. C. et al., 2017, ApJ, 838, 73

Massey P., Gronwall C., 1990, ApJ, 358, 344

McGovern M. R., Kirkpatrick J. D., McLean I. S., Burgasser A. J., Prato L., Lowrance P. J., 2004, ApJ, 600, 1020

Melis C., Reid M. J., Mioduszewski A. J., Stauffer J. R., Bower G. C., 2014, Science, 345, 1029

Oke J. B. et al., 1995, PASP, 107, 375

Ortega V. G., Jilinski E., de La Reza R., Bazzanella B., 2007, MNRAS, 377, 441

Pavlenko Y., Zapatero Osorio M. R., Rebolo R., 2000, A\&A, 355, 245

Pecaut M. J., Mamajek E. E., 2016, MNRAS, 461, 794

Pecaut M. J., Mamajek E. E., Bubar E. J., 2012, ApJ, 746, 154

Peña Ramírez K., Béjar V. J. S., Zapatero Osorio M. R., Petr-Gotzens M. G., Martín E. L., 2012, ApJ, 754, 30

Pérez-Garrido A., Lodieu N., Rebolo R., 2017, A\&A, 599, A78

Preibisch T., Brown A. G. A., Bridges T., Guenther E., Zinnecker H., 2002, AJ, 124, 404

Rajan A. et al., 2017, AJ, 154, 10

Reid I. N., Lewitus E., Allen P. R., Cruz K. L., Burgasser A. J., 2006, AJ, 132,891

Rich E. A., Currie T., Wisniewski J. P., Hashimoto J., Brandt T. D., Carson J. C., Kuzuhara M., Uyama T., 2016, ApJ, 830, 114

Sahlmann J., Lazorenko P. F., Bouy H., Martín E. L., Queloz D., Ségransan D., Zapatero Osorio M. R., 2016, MNRAS, 455, 357

Sarro L. M. et al., 2014, A\&A, 563, A45

Saumon D., Marley M. S., 2008, ApJ, 689, 1327

Schneider A. C., Windsor J., Cushing M. C., Kirkpatrick J. D., Shkolnik E. L., 2017, AJ, 153, 196

Simons D. A., Tokunaga A., 2002, PASP, 114, 169

Skemer A. J. et al., 2014, ApJ, 792, 17

Soderblom D. R., Nelan E., Benedict G. F., McArthur B., Ramirez I., Spiesman W., Jones B. F., 2005, AJ, 129, 1616

Soderblom D. R., Laskar T., Valenti J. A., Stauffer J. R., Rebull L. M., 2009, AJ, 138, 1292

Song I., Zuckerman B., Bessell M. S., 2012, AJ, 144, 8

Stauffer J. R., Schultz G., Kirkpatrick J. D., 1998, ApJ, 499, L199

Stephens D. C., Leggett S. K., 2004, PASP, 116, 9

Stone J. M. et al., 2016, ApJ, 818, L12

Takeda Y., Hashimoto O., Honda S., 2017, PASJ, 69, 1

Todorov K., Luhman K. L., McLeod K. K., 2010, ApJ, 714, L84

Tokunaga A. T., Simons D. A., Vacca W. D., 2002, PASP, 114, 180

Tremblin P., Amundsen D. S., Chabrier G., Baraffe I., Drummond B., Hinkley S., Mourier P., Venot O., 2016, ApJ, 817, L19
Vos J. M., Allers K. N., Biller B. A., 2017, ApJ, 842, 78

Vrba F. J. et al., 2004, AJ, 127, 2948

Yang H. et al., 2015, ApJ, 798, L13

Zapatero Osorio M. R. et al., 2014a, A\&A, 568, A77

Zapatero Osorio M. R. et al., 2014b, A\&A, 572, A67

Zapatero Osorio M. R., Béjar V. J. S., Peña Ramírez K., 2017, ApJ, 842, 65

\section{APPENDIX: FOLLOW-UP DATA OF NPMO3}

The new proper motion determination of NPM 03 is shown in Fig. 2. It agrees with that of Zapatero Osorio et al. (2014a) within $1 \sigma$ the quoted astrometric uncertainty. The Z-band magnitude (Table 2) indicates that this object is significantly bluer than Pleiades candidate members Calar 21, 22, and 24 despite having similar $J$-band brightness. In the discovery paper, and prior to the obtention of the data presented here, we speculated that it could be a metal-deficient ultracool dwarf or a white dwarf. The OSIRIS spectrum of NPM 03 shown in Fig. A1 clearly indicates that it is not an $\mathrm{M}$ or $\mathrm{L}$ or $\mathrm{T}$ dwarf. The optical spectrum peaks at around $5600 \AA$. The complete energy distribution (constructed as indicated in Section 4.3) suggests that NPM 03 is a white dwarf.

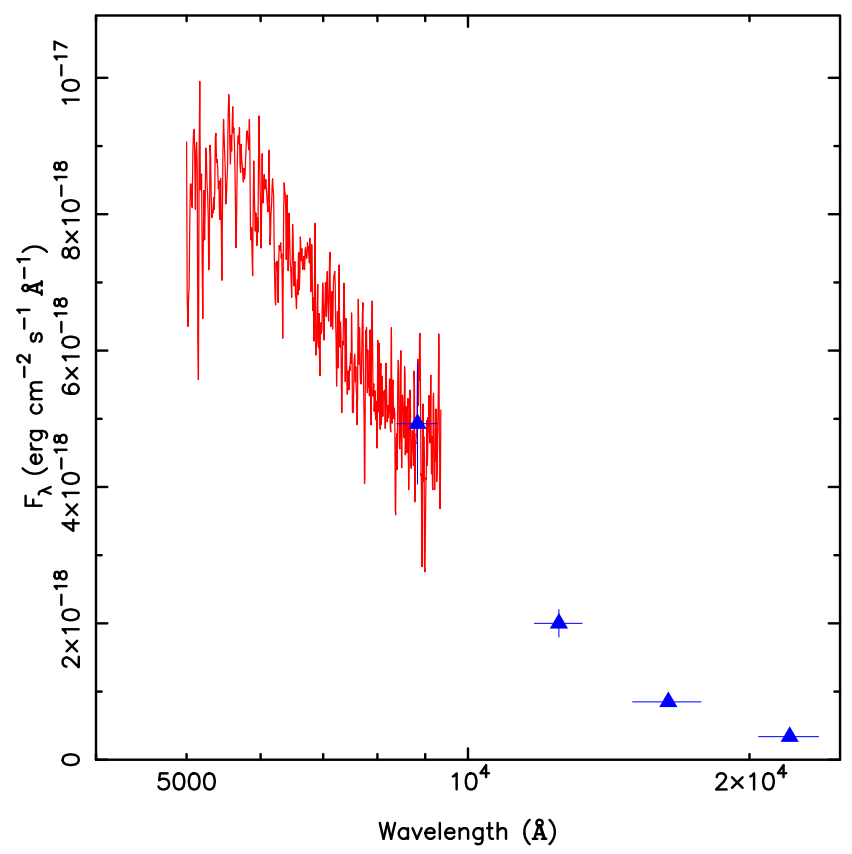

Figure A1. Observed spectrophotometric energy distribution of NPM03. The optical spectrum (red line) and the Z-band photometry (blue triangle overlapping with the optical spectrum) are presented here for the first time. The optical spectrum was flux calibrated using the Z-band photometry. The horizontal error bars associated with the photometry (blue triangles) indicate the passband of the filters. Near-infrared $J H K$ photometry comes from Zapatero Osorio et al. (2014a). The $x$-axis (wavelength) is shown in logarithmic scale.

This paper has been typeset from a $\mathrm{T}_{\mathrm{E}} \mathrm{X} / \mathrm{L} \mathrm{A} \mathrm{E} \mathrm{X}$ file prepared by the author. 